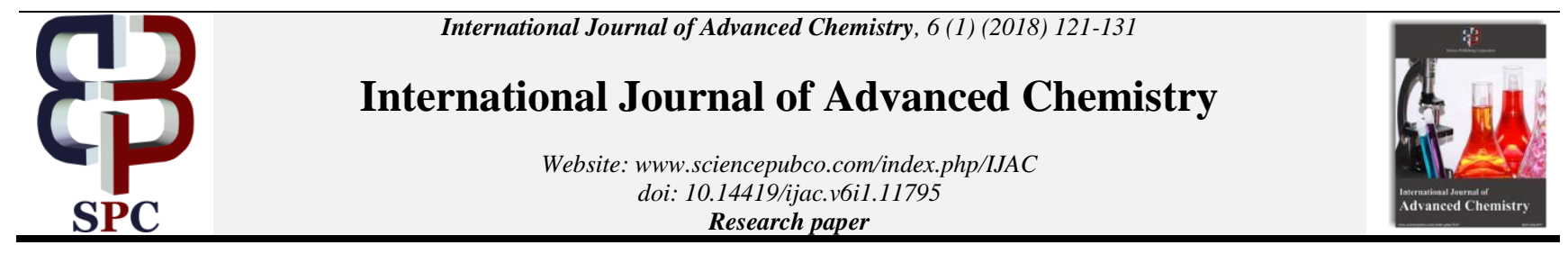

\title{
Quantum chemical investigation of spectroscopic, electronic and NLO properties of (1E, 4E)-1-(3-nitrophenyl)-5- phenylpenta-1,4-dien-3-one
}

\author{
N. Benhalima ${ }^{1}$, S. Yahiaoui ${ }^{1}$, N. Boubegra ${ }^{1}$, M. Boulakoud ${ }^{1}$, Y. Megrouss ${ }^{1}$, A. Chouaih ${ }^{1}$ * F. Hamzaoui $^{2}$ \\ ${ }^{1}$ LTPS Laboratory, Abdelhamid Ibn Badis University of Mostaganem, 27000 Mostaganem, Algeria \\ ${ }^{2}$ LPFM Académie de Montpellier - France \\ *Corresponding author E-mail: achouaih@gmail.com
}

\begin{abstract}
In the present work the optimized molecular geometry and harmonic vibrational frequencies of chalcone derivative were calculated by DFT/B3LYP method with 6-31G (d,p) basis set. The vibrational assignments were performed on the basis of the potential energy dis-tribution (PED) of the vibrational modes. Natural bond orbital (NBO) analysis has been performed on title compound using B3LYP/6-31G (d,p) and HSEh1PBE /6-31G (d,p) levels in order to elucidate intermolecular hydrogen bonding, intermolecular charge transfer (ICT) and delocalization of electron density. Mulliken atomic charges, natural population analysis (NPA) and atomic polar tensors (APT) were performed. The nonlinear optical properties of the title compound are also calculated and discussed. Molecular electrostatic poten-tial and HOMO-LUMO energy levels are also computed. Ultraviolet-visible spectrum of the title compound has been calculated using TD-DFT method. The molecular orbital contributions were studied by density of states (DOSs). Global reactivity descriptors have been calculated using the HOMO and LUMO to predict compound reactivity.
\end{abstract}

Keywords: DFT; HOMO; LUMO; NBO; NLO; NPA.

\section{Introduction}

In recent times, chalcone derivatives have received great attention due to their anti-infective, especially antifungal and antibacterial activities (Zangade et al. 2010, Abonia et al. 2012, Tala-Tapeh et al. 2015). It has reported that the potential biological activities of chalcones, such as, anti-inflammatory (Ballesteros et al. 1995, Won et al. 2005), antitumor (Kumar et al. 2003), antimalarial (Domínguez et al. 2005, Awasthi et al. 2009), inhibition of key enzymes (Rao et al. 2009), cytotoxicity (Reddy et al. 2012), antiplatelet (Zhao et al. 2005), antileishmanial (Aponte et al. 2010), antiviral (Biradar et al. 2010), inhibitor of colon cancer cell growth (Mizuno et al. 2010), Radical-scavenging (Nabi et al 2011), antidyslipidemic (Shukla et al. 2011), antidiabetic agents (Hsieh et al. 2012), vasorelaxant (Dong et al. 2010), antiprotozoal (Hayat et al. 2011) etc. In recent years, chalcones have been used in the field of material science as nonlinear optical (Shettigar et al. 2008, Asiri et al. 2014), optical limiting (Shettigar et al. 2006), electrochemical sensing (Delavaux-Nicot et al. 2007) and langmuir film (Gasull et al. 2002). Various materials containing chalcone moiety are notable for their second harmonic generation (Goto et al. 1991). The structure of the title compound was studied by the help of density functional theory (DFT) with B3LYP and HSEh1PBE functionals and 6-31G $(\mathrm{d}, \mathrm{p})$ basis set. The vibrational assignments have been performed based on potential energy distribution (PED). We have also performed natural bond orbital (NBO) calculation to provide a convenient basis for investigating charge transfer or conjugative interaction in molecular systems. Molecular electrostatic potential (MEP) surface and HOMO-
LUMO orbitals of the title compound have been investigated by using B3LYP and HSEh1PBE levels of density functional theory (DFT) with 6-31G (d,p) as basis set. In order to show the nonlinear optic (NLO) activity of title compound, the dipole moment, linear polarizability and first hyperpolarizability were obtained. Mulliken atomic charge, natural population analysis (NPA) and atomic polar tensors (APT) were computed by DFT method. The global reactivity descriptors, namely, hardness, softness, chemical potential and electrophilicity index are also calculated to understand the reactive nature of the compound.

\section{Computational details}

All theoretical calculations were carried out with help of Gaussian 09 program package (Frisch et al. 2009) using B3LYP (Becke's three parameter hybrid functional with the LYP correlation functional) (Lee et al. 1988, Becke et al. 1993) and HSEh1PBE (the recommended version of the full Heyd-Scuseria-Ernzerhof functional, referred to as HSE06 in literature) (Heyd et al. 2004, Heyd et al. 2004) methods in conjunction with 6-31G(d, p) basis set. The molecular structure, MEP surfaces and HOMO-LUMO levels were visualized with the help of Gauss View program (Frisch et al 2009). The theoretical vibrational spectra of the title compound were interpreted by means of PED using the VEDA 4 program (Jamroz et al. 2004). The calculated wavenumbers were scaled with scaling factor of 0.946 for B3LYP and 0.941 for HSEh1PBE. Natural bond orbital (NBO) calculations were performed using NBO 3.1 program (Glendening et al. 1998) as implemented in the Gaussian 09 package at the DFT/B3LYP and DFT/HSEh1PBE 
levels. The second order Fock-matrix was carried out to evaluate the donor (i) and acceptor (j) interaction in the NBO basis (Foster et al. 1980, Reed et al. 1983, Reed et al. 1985, Reed et al. 1985, Reed et al. 1988, Szafran et al. 2007, Sebastian et al. 2010, Tamer et al. 2015, Altürk et al. 2015). For each donor (i) and acceptor (j), the stabilization energy $\mathrm{E}^{(2)}$ associated as:

$E^{(2)}=q_{i} \frac{F(i, j)^{2}}{\varepsilon_{i}-\varepsilon_{j}}$

The larger the $\mathrm{E}^{(2)}$ value, the more intensive is the interaction between electron donor and electron acceptor, i.e. a more donating tendency from electron donors to acceptors and a greater extent of conjugation of the whole system. Ultraviolet-visible spectrum, excitation energies, absorbance and oscillator strengths for the title compound were obtained in the framework of TD-DFT (Cancès et al. 1997, Stratmann et al. 1998) calculations using B3LYP/6-31G (d,p) and HSEh1PBE/6-31G (d,p) functionals. Density of state (DOS) diagram of the title compound derived using the GaussSum 2.2 program (O'Boyle et al. 2008) was used to calculate group contributions to the molecular orbitals. Molecular electrostatic potential (MEP) surfaces have been investigated by using B3LYP and HSEh1PBE levels of DFT method with 6-31G $(d, p)$ basis set Mulliken atomic charges, natural population analysis (NPA) and atomic polar tensors (APT) (Ferreira 1993) were calculated by determining the electron population of each atom. To study the nonlinear optical properties (NLO), parameters such as dipole moment $(\mu)$, polarizability $(\alpha)$ and the first-order hyperpolarizability $(\beta)$ are determined using B3LYP and HSEh1PBE functionals with $6-31 \mathrm{G}(\mathrm{d}, \mathrm{p})$ basis sets. The total dipole moments $(\mu)$, polarizability $(\alpha)$ and first-order hyperpolarizability $(\beta)$ are evaluated using the following equations:

$\mu=\left(\mu_{\mathrm{x}}^{2}+\mu_{\mathrm{y}}^{2}+\mu_{\mathrm{z}}^{2}\right)^{1 / 2}$

$\alpha=\frac{1}{3}\left(\alpha_{\mathrm{x}}+\alpha_{\mathrm{y}}+\alpha_{\mathrm{z}}\right)$

$\beta_{\text {tot }}=\left(\beta_{\mathrm{x}}^{2}+\beta_{\mathrm{y}}^{2}+\beta_{\mathrm{z}}^{2}\right)^{1 / 2}$

The complete equation for calculating the magnitude of first-order hyper polarizability from Gaussian 09 output is given as follows:

$$
\begin{aligned}
& \beta_{\mathrm{tot}}=\left[\left(\beta_{\mathrm{xxx}}^{2}+\beta_{\mathrm{xyy}}^{2}+\beta_{\mathrm{xzz}}^{2}\right)^{2}+\left(\beta_{\mathrm{xxy}}^{2}+\beta_{\mathrm{yyy}}^{2}+\beta_{\mathrm{yzz}}^{2}\right)^{2}+\right. \\
& \left.\left(\beta_{\mathrm{xxz}}^{2}+\beta_{\mathrm{yyz}}^{2}+\beta_{\mathrm{zzz}}^{2}\right)^{2}\right]^{1 / 2}
\end{aligned}
$$

The $\alpha_{\text {tot }}$ and $\beta_{\text {tot }}$ values of Gaussian output are in atomic units (a.u.) therefore they were converted into electrostatic units (esu) (for $\alpha$; 1 a.u. $=0.1482 \times 10^{-24} \mathrm{esu}$, for $\beta ; 1$ a.u. $\left.=8.6393 \times 10^{-33} \mathrm{esu}\right)$. The chemical reactivity descriptors of compounds such as electronegativity $(\chi)$, chemical potential $(\mu)$, hardness $(\eta)$, softness (S), and electrophilicity index $(\omega)$ are evaluated using following equations (Parr et al. 1978, Parr et al. 1983, Parr et al. 1999, Chattaraj et al. 2003, Pearson 2005):

$$
\begin{aligned}
& \chi=\frac{I+A}{2} \\
& \mu=-\frac{I+A}{2} \\
& \eta=\frac{I-A}{2} \\
& S=\frac{1}{2} \eta \\
& \omega=\frac{\mu^{2}}{2 \eta}
\end{aligned}
$$

\section{Results and discussion}

\subsection{Optimized structure and hydrogen bonding}

The bond lengths, bond angles and dihedral angles corresponding to the optimized geometry of the title compound have been obtained using the DFT/B3LYP/6-31(d,p) and DFT/HSEh1PBE /6$31(\mathrm{~d}, \mathrm{p})$ methods and the corresponding results are reported in Table 1. The optimized geometry of the title compound is illustrated in Fig. 1. The benzene $\mathrm{C}-\mathrm{C}$ bond lengths observed in the region of 1.378-1.399 $\AA$ (Samshuddin et al. 2012) have been calculated at the range of $1.390-1.410 \AA$ for B3LYP level and 1.386$1.406 \AA$ for HSEh1PBE level. The $\mathrm{C} 7=\mathrm{C} 8$ and $\mathrm{C} 10=\mathrm{C} 11$ bond lengths are observed as 1.332, $1.333 \AA$ (Samshuddin et al. 2012). In our calculations, these bond length are found to be 1.346 , $1.346 \AA$ for B3LYP level and 1.343, $1.346 \AA$ for HSEh1PBE level. For the title compound, the $\mathrm{C}=\mathrm{O}$ bond lengths (B3LYP /XRD) are 1.2308/1.2287 $\AA$ which are in agreement with reported values (Samshuddin et al. 2012). The bond length between $C$ and $\mathrm{N}$ is $0,001 \AA$ times higher than the experimental value $(1.465 \AA)$. The bond length of $\mathrm{O}-\mathrm{N}$ is differed from $0.003 \AA$ to $0.01 \AA$ with experimental value (1.2270 and 1.2202 $\AA$ ). The average ON distance of $1.2301 \AA$ in the nitro group is an indication of clearly double bonds. The bond angle $\mathrm{O} 1-\mathrm{N} 1-\mathrm{O} 2\left(124.78^{\circ}\right)$ of nitro group which is closer to experimental data $\left(123.25^{\circ}\right)$. The calculated $\mathrm{O} 1-\mathrm{N} 1-\mathrm{C} 3$ and $\mathrm{O} 2-\mathrm{N} 1-\mathrm{C} 3$ angles are 117.68 and 117.54, respectively, about $0.7^{\circ}$ smaller than the experimental results. The N1-C3-C2 and N1-C3-C4 bond angles observed as $118.73^{\circ}$ and $118.61^{\circ}$ (Samshuddin et al. 2012) have been also calculated as 118.79 and 118.83 for B3LYP, 118.75 and 118.80 for HSEh1PBE levels. From Table 1, it can be noted that there is a good agreement between the experimental and theoretical geometric parameters.

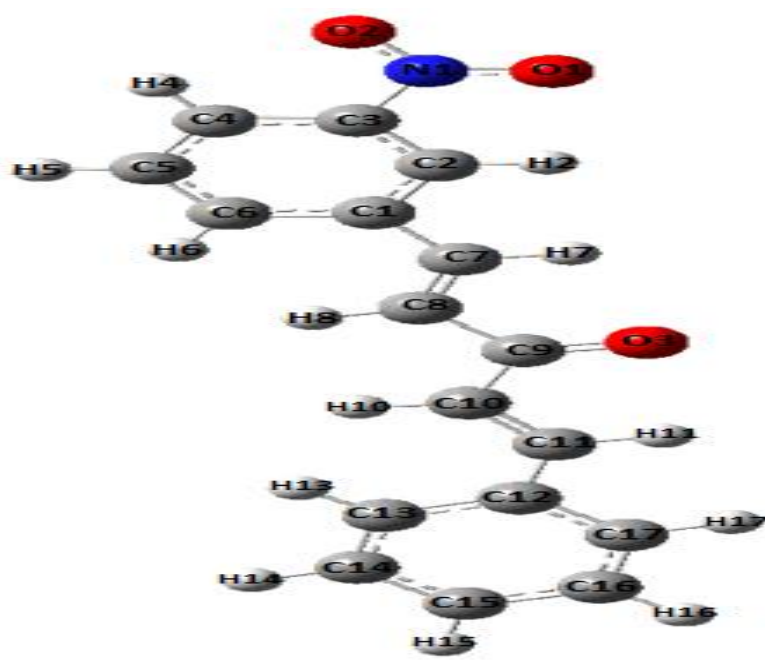

Fig. 1: The Optimized Structure of the Title Compound

\subsection{Natural bond orbital analysis}

The natural bond orbital analysis have been performed by using B3LYP and HSEh1PBE methods with 6-31G (d, p) basis set. The perturbation energies of significant donor-acceptor interactions from the NBO analysis were summarized in Table 2 . As concluded from the calculated NBO results, the strongest stabilization energies within title compound are defined as $\pi(\mathrm{C} 7-\mathrm{C} 8) \rightarrow \pi^{*}$ (C9-O3) with the energies of 19.32 and $18.84 \mathrm{kcal} / \mathrm{mol}$ at B3LYP and HSEh1PBE levels, respectively. The important intramolecular hyper-conjugative interactions are: $\mathrm{n} 2(\mathrm{O} 1) \rightarrow \sigma^{*}(\mathrm{C} 3-\mathrm{N} 1)$, n2 $(\mathrm{O} 1) \rightarrow \sigma^{*}(\mathrm{~N} 1-\mathrm{O} 2), \mathrm{n} 2(\mathrm{O} 2) \rightarrow \sigma^{*}(\mathrm{C} 3-\mathrm{N} 1), \mathrm{n} 2(\mathrm{O} 2) \rightarrow \sigma^{*}(\mathrm{~N} 1-$ $\mathrm{O} 1), \mathrm{n} 3(\mathrm{O} 2) \rightarrow(\mathrm{N} 1-\mathrm{O} 1), \mathrm{n} 2(\mathrm{O} 3) \rightarrow \sigma^{*}(\mathrm{C} 8-\mathrm{C} 9), \mathrm{n} 2(\mathrm{O} 3) \rightarrow \sigma^{*}$ (C9-C10)with stabilization energies $12.84,19.30,12.77,19.22$, 164.16, 19.79, $19.17 \mathrm{~kJ} / \mathrm{mol}$ at B3LYP . The corresponding electron density values are $1.89710 \mathrm{e}, 1.89771 \mathrm{e}, 1.44242 \mathrm{e}$ and 
1.88050e. The magnitude of charge transfer from the lone pairs of $\mathrm{n} 3(\mathrm{O} 2)$ to anti-bonding (N1-O1), $\pi$ orbitals amount to stabilization of $164.16 \mathrm{kcal} / \mathrm{mol}$. The highly probable transition in this compound are $(\mathrm{C} 1-\mathrm{C} 2)$ to $(\mathrm{C} 3-\mathrm{C} 4)\left(\pi-\pi^{*}, 20.56 \mathrm{kcal} / \mathrm{mol}\right),(\mathrm{C} 1-$ $\mathrm{C} 2)$ to (C5-C6) $\left(\pi \rightarrow \pi^{*}, 20.01 \mathrm{kcal} / \mathrm{mol}\right),(\mathrm{C} 3-\mathrm{C} 4)$ to $(\mathrm{C} 1-\mathrm{C} 2)$ $\left(\pi \rightarrow \pi^{*}, 21.60 \mathrm{kcal} / \mathrm{mol}\right), \quad(\mathrm{C} 3-\mathrm{C} 4)$ to $(\mathrm{C} 5-\mathrm{C} 6)\left(\pi \rightarrow \pi^{*}, 16.48\right.$ $\mathrm{kcal} / \mathrm{mol}),(\mathrm{C} 5-\mathrm{C} 6)$ to $(\mathrm{C} 1-\mathrm{C} 2)\left(\pi \rightarrow \pi^{*}, 18.23 \mathrm{kcal} / \mathrm{mol}\right),(\mathrm{C} 5-\mathrm{C} 6)$ to $(\mathrm{C} 3-\mathrm{C} 4) \quad\left(\pi \rightarrow \pi^{*}, 22.60 \mathrm{kcal} / \mathrm{mol}\right)$, these six transitions corre- spond to three pairs of orbitals within the first aromatic ring. $\mathrm{E}^{(2)}$ means energy of hyperconjugative interactions (stabilization energy).

An Energy difference between donor and acceptor $\mathrm{i}$ and $\mathrm{j}$ NBO orbitals.

${ }^{B} F(i, j)$ is the Fock matrix element between $i$ and $j \mathrm{NBO}$ orbitals.

Table 1: The Calculated Geometric Parameters of the Title Compound

\begin{tabular}{|c|c|c|c|c|c|c|c|c|}
\hline Bond lengths $(\AA)$ & X-ray & DFT & Bond angles $\left({ }^{\circ}\right)$ & $\mathrm{X}$-ray & DFT & Dihedral angles $\left({ }^{\circ}\right)$ & X-ray & DFT \\
\hline $\mathrm{O} 1-\mathrm{N} 1$ & 1.227 & 1.229 & O1-N1-O2 & 123.25 & 124.78 & O1-N1-C3-C2 & 6.3 & 0.0 \\
\hline $\mathrm{O} 2-\mathrm{N} 1$ & 1.220 & 1.230 & $\mathrm{O} 1-\mathrm{N} 1-\mathrm{C} 3$ & 118.38 & 117.68 & O1-N1-C3-C4 & -172.4 & -179.9 \\
\hline $\mathrm{O} 3-\mathrm{C} 9$ & 1.228 & 1.230 & $\mathrm{O} 2-\mathrm{N} 1-\mathrm{C} 3$ & 118.36 & 117.54 & $\mathrm{O} 2-\mathrm{N} 1-\mathrm{C} 3-\mathrm{C} 2$ & -173.8 & -179.9 \\
\hline $\mathrm{N} 1-\mathrm{C} 3$ & 1.465 & 1.475 & $\mathrm{C} 2-\mathrm{C} 1-\mathrm{C} 6$ & 118.32 & 118.12 & O2-N1-C3-C4 & 7.4 & 0.0 \\
\hline $\mathrm{C} 1-\mathrm{C} 2$ & 1.394 & 1.404 & $\mathrm{C} 2-\mathrm{C} 1-\mathrm{C} 7$ & 118.78 & 118.43 & $\mathrm{C} 6-\mathrm{C} 1-\mathrm{C} 2-\mathrm{C} 3$ & -1.6 & -0.0 \\
\hline $\mathrm{C} 1-\mathrm{C} 6$ & 1.399 & 1.410 & $\mathrm{C} 6-\mathrm{C} 1-\mathrm{C} 7$ & 122.89 & 123.45 & $\mathrm{C} 7-\mathrm{C} 1-\mathrm{C} 2-\mathrm{C} 3$ & 177.6 & 180.0 \\
\hline $\mathrm{C} 1-\mathrm{C} 7$ & 1.467 & 1.464 & $\mathrm{C} 1-\mathrm{C} 2-\mathrm{C} 3$ & 119.22 & 119.69 & $\mathrm{C} 2-\mathrm{C} 1-\mathrm{C} 6-\mathrm{C} 5$ & 0.6 & 0.0 \\
\hline $\mathrm{C} 2-\mathrm{C} 3$ & 1.384 & 1.390 & $\mathrm{~N} 1-\mathrm{C} 3-\mathrm{C} 2$ & 118.73 & 118.79 & C7-C1-C6-C5 & -178.6 & -180.0 \\
\hline C3-C4 & 1.383 & 1.392 & $\mathrm{~N} 1-\mathrm{C} 3-\mathrm{C} 4$ & 118.61 & 118.83 & $\mathrm{C} 2-\mathrm{C} 1-\mathrm{C} 7-\mathrm{C} 8$ & -173.2 & -179.9 \\
\hline C4-C5 & 1.385 & 1.395 & $\mathrm{C} 2-\mathrm{C} 3-\mathrm{C} 4$ & 122.65 & 122.37 & $\mathrm{C} 6-\mathrm{C} 1-\mathrm{C} 7-\mathrm{C} 8$ & 6.1 & 0.0 \\
\hline C5-C6 & 1.383 & 1.392 & C3-C4-C5 & 118.09 & 118.06 & $\mathrm{C} 1-\mathrm{C} 2-\mathrm{C} 3-\mathrm{N} 1$ & -177.1 & -180.0 \\
\hline C7-C8 & 1.332 & 1.346 & C4-C5-C6 & 120.27 & 120.54 & $\mathrm{C} 1-\mathrm{C} 2-\mathrm{C} 3-\mathrm{C} 4$ & 1.5 & 0.0 \\
\hline C8-C9 & 1.482 & 1.489 & $\mathrm{C} 1-\mathrm{C} 6-\mathrm{C} 5$ & 121.43 & 121.22 & $\mathrm{~N} 1-\mathrm{C} 3-\mathrm{C} 4-\mathrm{C} 5$ & 178.4 & -180.0 \\
\hline C9-C10 & 1.479 & 1.481 & $\mathrm{C} 1-\mathrm{C} 7-\mathrm{C} 8$ & 126.52 & 127.86 & $\mathrm{C} 2-\mathrm{C} 3-\mathrm{C} 4-\mathrm{C} 5$ & -0.2 & -0.0 \\
\hline C10-C11 & 1.333 & 1.346 & $\mathrm{C} 7-\mathrm{C} 8-\mathrm{C} 9$ & 122.11 & 120.49 & C3-C4-C5-C6 & -0.8 & 0.0 \\
\hline $\mathrm{C} 11-\mathrm{C} 12$ & 1.468 & 1.461 & $\mathrm{O} 3-\mathrm{C} 9-\mathrm{C} 8$ & 122.43 & 121.46 & $\mathrm{C} 1-\mathrm{C} 7-\mathrm{C} 8-\mathrm{C} 9$ & -177.4 & 180.0 \\
\hline $\mathrm{C} 12-\mathrm{C} 13$ & 1.395 & 1.409 & $\mathrm{O} 3-\mathrm{C} 9-\mathrm{C} 10$ & 122.50 & 122.34 & $\mathrm{C} 7-\mathrm{C} 8-\mathrm{C} 9-\mathrm{O} 3$ & -11.3 & 0.0 \\
\hline C12-C17 & 1.397 & 1.407 & C8-C9-C10 & 115.02 & 116.19 & C7-C8-C9-C10 & 166.1 & -180.0 \\
\hline C13-C14 & 1.387 & 1.409 & C9-C10-C11 & 121.79 & 120.56 & O3-C9-C10-C11 & 21.9 & 0.0 \\
\hline C14-C15 & 1.386 & 1.399 & $\mathrm{C} 10-\mathrm{C} 11-\mathrm{C} 12$ & 126.80 & 128.29 & C8-C9-C10-C11 & -155.4 & -180.0 \\
\hline C15-C16 & 1.378 & 1.395 & $\mathrm{C} 11-\mathrm{C} 12-\mathrm{C} 13$ & 122.53 & 123.34 & C9-C10-C11-C12 & 176.1 & 180. \\
\hline \multirow[t]{9}{*}{ C16-C17 } & 1.389 & 1.393 & C11-C12-C17 & 118.99 & 118.49 & $\mathrm{C} 10-\mathrm{C} 11-\mathrm{C} 12-\mathrm{C} 13$ & 10.4 & 0.0 \\
\hline & & & $\mathrm{C} 13-\mathrm{C} 12-\mathrm{C} 17$ & 118.46 & 118.18 & $\mathrm{C} 10-\mathrm{C} 11-\mathrm{C} 12-\mathrm{C} 17$ & -170.9 & -180.0 \\
\hline & & & $\mathrm{C} 12-\mathrm{C} 13-\mathrm{C} 14$ & 120.55 & 120.73 & C11-C12-C13-C14 & 178.3 & -180.0 \\
\hline & & & C13-C14-C15 & 120.36 & 120.31 & $\mathrm{C} 17-\mathrm{C} 12-\mathrm{C} 13-\mathrm{C} 14$ & -0.3 & 0.0 \\
\hline & & & C14-C15-C16 & 119.65 & 119.74 & C11-C12-C17-C16 & -177.9 & 180.0 \\
\hline & & & C15-C16-C17 & 120.37 & 119.94 & C13-C12-C17-C16 & 0.8 & -0.0 \\
\hline & & & C12-C17-C16 & 120.61 & 121.10 & C12-C13-C14-C15 & -0.3 & -0.0 \\
\hline & & & & & & C13-C14-C15-C16 & 0.4 & 0.0 \\
\hline & & & & & & $\mathrm{C} 14-\mathrm{C} 15-\mathrm{C} 16-\mathrm{C} 17$ & 0.0 & -0.0 \\
\hline
\end{tabular}

Table.2: Second-Order Perturbation Theory Analysis of Flock Matrix in NBO Basis Corresponding to the Intra-Molecular Bonds of the Title Compound.

\begin{tabular}{|c|c|c|c|c|c|c|}
\hline Donor (i) & ED (i) (e) & Acceptor (j) & ED (j) (e) & $\begin{array}{l}\mathrm{E}^{(2)} \\
\left(\mathrm{kcal} \mathrm{mol}^{1}\right)\end{array}$ & $\varepsilon_{\mathrm{j}} \varepsilon_{\mathrm{i}}^{\mathrm{a}}$ (a.u.) & $\mathrm{F}(\mathrm{i}, \mathrm{j})^{\mathrm{b}}(\mathrm{a} . \mathrm{u})$ \\
\hline$\pi(\mathrm{C} 1-\mathrm{C} 2)$ & 1.60465 & $\pi^{*}(\mathrm{C} 3-\mathrm{C} 4)$ & 0.37340 & 20.56 & 0.27 & 0.067 \\
\hline$\pi(\mathrm{C} 1-\mathrm{C} 2)$ & & $\pi^{*}(\mathrm{C} 5-\mathrm{C} 6)$ & 0.28810 & 20.01 & 0.28 & 0.069 \\
\hline$\pi(\mathrm{C} 1-\mathrm{C} 2)$ & & $\pi^{*}(\mathrm{C} 7-\mathrm{C} 8)$ & 0.09427 & 15.49 & 0.30 & 0.066 \\
\hline$\pi(\mathrm{C} 3-\mathrm{C} 4)$ & 1.64122 & $\pi^{*}(\mathrm{C} 1-\mathrm{C} 2)$ & 0.34153 & 21.60 & 0.30 & 0.072 \\
\hline$\pi(\mathrm{C} 3-\mathrm{C} 4)$ & & $\pi^{*}(\mathrm{C} 5-\mathrm{C} 6)$ & 0.28810 & 16.48 & 0.29 & 0.063 \\
\hline$\pi(\mathrm{C} 3-\mathrm{C} 4)$ & & $\pi^{*}(\mathrm{~N} 1-\mathrm{O} 1)$ & 0.62218 & 27.22 & 0.15 & 0.061 \\
\hline$\pi(\mathrm{C} 5-\mathrm{C} 6)$ & 1.65472 & $\pi^{*}(\mathrm{C} 1-\mathrm{C} 2)$ & 0.34153 & 18.23 & 0.29 & 0.064 \\
\hline$\pi(\mathrm{C} 5-\mathrm{C} 6)$ & & $\pi^{*}(\mathrm{C} 3-\mathrm{C} 4)$ & 0.37340 & 22.60 & 0.28 & 0.071 \\
\hline$\pi(\mathrm{C} 7-\mathrm{C} 8)$ & 1.84165 & $\pi^{*}(\mathrm{C} 1-\mathrm{C} 2)$ & 0.34153 & 12.29 & 0.29 & 0.056 \\
\hline$\pi(\mathrm{C} 7-\mathrm{C} 8)$ & & $\pi^{*}(\mathrm{C} 9-\mathrm{O} 3)$ & 0.23046 & 19.32 & 0.30 & 0.069 \\
\hline$\pi(\mathrm{C} 10-\mathrm{C} 11)$ & 1.83189 & $\pi^{*}(\mathrm{C} 9-\mathrm{O} 3)$ & 0.23046 & 21.62 & 0.29 & 0.072 \\
\hline$\pi(\mathrm{C} 10-\mathrm{C} 11)$ & & $\pi^{*}(\mathrm{C} 12-\mathrm{C} 17)$ & 0.37438 & 11.59 & 0.30 & 0.055 \\
\hline$\pi(\mathrm{C} 12-\mathrm{C} 17)$ & 1.61495 & $\pi^{*}(\mathrm{C} 10-\mathrm{C} 11)$ & 0.10872 & 17.10 & 0.29 & 0.068 \\
\hline$\pi(\mathrm{C} 12-\mathrm{C} 17)$ & & $\pi^{*}(\mathrm{C} 13-\mathrm{C} 14)$ & 0.29620 & 19.18 & 0.28 & 0.067 \\
\hline$\pi(\mathrm{C} 12-\mathrm{C} 17)$ & & $\pi^{*}(\mathrm{C} 15-\mathrm{C} 16)$ & 0.32266 & 19.60 & 0.28 & 0.067 \\
\hline$\pi(\mathrm{C} 13-\mathrm{C} 14)$ & 1.67848 & $\pi^{*}(\mathrm{C} 12-\mathrm{C} 17)$ & 0.37438 & 18.85 & 0.28 & 0.066 \\
\hline$\pi(\mathrm{C} 13-\mathrm{C} 14)$ & & $\pi^{*}(\mathrm{C} 15-\mathrm{C} 16)$ & 0.32266 & 20.22 & 0.28 & 0.068 \\
\hline$\pi(\mathrm{C} 15-\mathrm{C} 16)$ & 1.65023 & $\pi^{*}(\mathrm{C} 12-\mathrm{C} 17)$ & 0.37438 & 21.18 & 0.28 & 0.069 \\
\hline$\pi(\mathrm{C} 15-\mathrm{C} 16)$ & & $\pi^{*}(\mathrm{C} 13-\mathrm{C} 14)$ & 0.29620 & 18.97 & 0.28 & 0.066 \\
\hline$\pi(\mathrm{N} 1-\mathrm{O} 1)$ & 1.98600 & $\mathrm{n} 3(\mathrm{O} 2)$ & 1.44242 & 12.35 & 0.18 & 0.078 \\
\hline $\mathrm{n} 2(\mathrm{O} 1)$ & 1.89710 & $\sigma^{*}(\mathrm{C} 3-\mathrm{N} 1)$ & 0.10609 & 12.84 & 0.57 & 0.076 \\
\hline n2 (O1) & & $\sigma^{*}(\mathrm{~N} 1-\mathrm{O} 2)$ & 0.05664 & 19.30 & 0.71 & 0.105 \\
\hline n2 (O2) & 1.89771 & $\sigma^{*}(\mathrm{C} 3-\mathrm{N} 1)$ & 0.10609 & 12.77 & 0.57 & 0.076 \\
\hline n2 (O2) & & $\sigma^{*}(\mathrm{~N} 1-\mathrm{O} 1)$ & 0.05650 & 19.22 & 0.71 & 0.105 \\
\hline n3 (O2) & 1.44242 & $\pi^{*}(\mathrm{~N} 1-\mathrm{O} 1)$ & 0.62218 & 164.16 & 0.14 & 0.139 \\
\hline n2 (O3) & 1.88050 & $\sigma^{*}(\mathrm{C} 8-\mathrm{C} 9)$ & 0.06002 & 19.79 & 0.69 & 0.106 \\
\hline n2 (O3) & & $\sigma^{*}(\mathrm{C} 9-\mathrm{C} 10)$ & 0.05765 & 19.17 & 0.70 & 0.105 \\
\hline$\pi^{*}(\mathrm{C} 1-\mathrm{C} 2)$ & 0.34153 & $\pi^{*}(\mathrm{C} 7-\mathrm{C} 8)$ & 0.09427 & 47.80 & 0.02 & 0.064 \\
\hline$\pi^{*}(\mathrm{C} 9-\mathrm{O} 3)$ & 0.23046 & $\pi^{*}(\mathrm{C} 7-\mathrm{C} 8)$ & 0.09427 & 67.07 & 0.02 & 0.072 \\
\hline$\pi * \mathrm{C} 9-\mathrm{O} 3)$ & & $\pi^{*}(\mathrm{C} 10-\mathrm{C} 11)$ & 0.10872 & 53.10 & 0.02 & 0.072 \\
\hline$\pi^{*}(\mathrm{C} 12-\mathrm{C} 17)$ & 0.37438 & $\pi^{*}(\mathrm{C} 10-\mathrm{C} 11)$ & 0.10872 & 107.46 & 0.01 & 0.067 \\
\hline$\pi^{*}(\mathrm{~N} 1-\mathrm{O} 1)$ & 0.62218 & $\pi^{*}(\mathrm{C} 3-\mathrm{C} 4)$ & 0.37340 & 15.69 & 0.14 & 0.059 \\
\hline
\end{tabular}


Table 3: Selected NBO Results Showing Formation of Lewis and Non- Lewis Orbitals of the Title Compound Using B3LYP/631G (D, P) Level of Theo-

\begin{tabular}{|c|c|c|c|c|c|c|c|}
\hline Bond $(\mathrm{AB})$ & ED/energy(a.u.) & $\operatorname{EDA}(\%)$ & & EDB (\%) & $\mathrm{NBO}$ & $\mathrm{S}(\%)$ & $\mathrm{p}(\%)$ \\
\hline$\Sigma(\mathrm{C} 1-\mathrm{C} 2)$ & 1.97144 & 50.65 & & 49.35 & $0.7117\left(\mathrm{sp}^{2.01}\right) \mathrm{C}+0.7025\left(\mathrm{sp}^{1.00}\right) \mathrm{C}$ & $\begin{array}{l}33.26 \\
35.41\end{array}$ & $\begin{array}{l}66.70 \\
64.55\end{array}$ \\
\hline$\Sigma(\mathrm{C} 1-\mathrm{C} 6)$ & 1.97586 & 51.04 & & 48.96 & $0.7144\left(\mathrm{sp}^{1.95}\right) \mathrm{C}+0.6997\left(\mathrm{sp}^{1.88}\right) \mathrm{C}$ & $\begin{array}{l}33.87 \\
34.73\end{array}$ & $\begin{array}{l}66.09 \\
65.23\end{array}$ \\
\hline$\Sigma(\mathrm{C} 1-\mathrm{C} 7)$ & 1.97679 & 51.52 & & 48.48 & $0.7178\left(\mathrm{sp}^{2.05}\right) \mathrm{C}+0.6963\left(\mathrm{sp}^{1.96}\right) \mathrm{C}$ & $\begin{array}{l}32.82 \\
33.75\end{array}$ & $\begin{array}{l}67.14 \\
66.20\end{array}$ \\
\hline$\Sigma(\mathrm{C} 2-\mathrm{C} 3)$ & 1.97644 & 49.06 & & 50.94 & $0.7004\left(\mathrm{sp}^{1.94}\right) \mathrm{C}+0.7137\left(\mathrm{sp}^{1.64}\right) \mathrm{C}$ & $\begin{array}{l}34.05 \\
37.90\end{array}$ & $\begin{array}{l}65.90 \\
62.07\end{array}$ \\
\hline$\Sigma(\mathrm{C} 3-\mathrm{C} 4)$ & 1.97665 & 51.15 & & 48.85 & $0.7152\left(\mathrm{sp}^{1.65}\right) \mathrm{C}+0.6989\left(\mathrm{sp}^{1.97}\right) \mathrm{C}$ & $\begin{array}{l}37.77 \\
33.61\end{array}$ & $\begin{array}{l}62.19 \\
66.34\end{array}$ \\
\hline$\Sigma(\mathrm{C} 3-\mathrm{N} 1)$ & 1.98966 & 37.82 & & 62.18 & $0.6150\left(\mathrm{sp}^{3.12}\right) \mathrm{C}+0.7885\left(\mathrm{sp}^{1.77}\right) \mathrm{N}$ & $\begin{array}{l}24.26 \\
36.08\end{array}$ & $\begin{array}{l}75.61 \\
63.88\end{array}$ \\
\hline$\Sigma(\mathrm{C} 4-\mathrm{C} 5)$ & 1.97626 & 50.37 & & 49.63 & $0.7097\left(\mathrm{sp}^{1.83}\right) \mathrm{C}+0.7045\left(\mathrm{sp}^{1.88}\right) \mathrm{C}$ & $\begin{array}{l}35.38 \\
34.76\end{array}$ & $\begin{array}{l}64.59 \\
65.20\end{array}$ \\
\hline$\Sigma(\mathrm{C} 5-\mathrm{C} 6)$ & 1.98121 & 49.84 & & 50.16 & $0.7060\left(\mathrm{sp}^{1.83}\right) \mathrm{C}+0.7082\left(\mathrm{sp}^{1.80}\right) \mathrm{C}$ & $\begin{array}{l}35.27 \\
35.70\end{array}$ & $\begin{array}{l}64.69 \\
64.27\end{array}$ \\
\hline$\Sigma(\mathrm{C} 7-\mathrm{C} 8)$ & 1.98316 & 50.33 & & 49.67 & $0.7094\left(\mathrm{sp}^{1.64}\right) \mathrm{C}+0.7048\left(\mathrm{sp}^{1.65}\right) \mathrm{C}$ & $\begin{array}{l}37.86 \\
37.79\end{array}$ & $\begin{array}{l}62.11 \\
62.18\end{array}$ \\
\hline$\Sigma(\mathrm{C} 8-\mathrm{C} 9)$ & 1.98075 & 51.60 & & 48.40 & $0.7183\left(\mathrm{sp}^{2.08}\right) \mathrm{C}+0.6957\left(\mathrm{sp}^{1.91}\right) \mathrm{C}$ & $\begin{array}{l}32.41 \\
34.38\end{array}$ & $\begin{array}{l}67.54 \\
65.57\end{array}$ \\
\hline$\Sigma(\mathrm{C} 9-\mathrm{C} 10)$ & 1.98122 & 48.72 & & 51.28 & $0.6980\left(\mathrm{sp}^{1.84}\right) \mathrm{C}+0.7161\left(\mathrm{sp}^{2.08}\right) \mathrm{C}$ & $\begin{array}{l}35.24 \\
32.43\end{array}$ & $\begin{array}{l}64.71 \\
67.53\end{array}$ \\
\hline$\Sigma(\mathrm{C} 9-\mathrm{O} 3)$ & 1.99534 & 34.66 & & 65.34 & $0.5888\left(\mathrm{sp}^{2.30}\right) \mathrm{C}+0.8083\left(\mathrm{sp}^{1.39}\right) \mathrm{O}$ & $\begin{array}{l}30.30 \\
41.76\end{array}$ & $\begin{array}{l}69.59 \\
57.91\end{array}$ \\
\hline$\Sigma(\mathrm{C} 10-\mathrm{C} 11)$ & 1.98305 & 49.79 & & 50.21 & $0.7056\left(\mathrm{sp}^{1.65}\right) \mathrm{C}+0.7086\left(\mathrm{sp}^{1.65}\right) \mathrm{C}$ & $\begin{array}{l}37.73 \\
37.69\end{array}$ & $\begin{array}{l}62.23 \\
62.27\end{array}$ \\
\hline$\Sigma(\mathrm{C} 11-\mathrm{C} 12)$ & 1.97734 & 48.94 & & 51.06 & $0.6995\left(\mathrm{sp}^{1.92}\right) \mathrm{C}+0.7146\left(\mathrm{sp}^{2.08}\right) \mathrm{C}$ & $\begin{array}{l}34.29 \\
32.42\end{array}$ & $\begin{array}{l}65.67 \\
67.54\end{array}$ \\
\hline$\Sigma(\mathrm{C} 12-\mathrm{C} 13)$ & 1.97486 & 51.08 & & 48.92 & $0.7147\left(\mathrm{sp}^{1.94}\right) \mathrm{C}+0.6994\left(\mathrm{sp}^{1.88}\right) \mathrm{C}$ & $\begin{array}{l}33.97 \\
34.71\end{array}$ & $\begin{array}{l}65.99 \\
65.25\end{array}$ \\
\hline$\Sigma(\mathrm{C} 12-\mathrm{C} 17)$ & 1.97555 & 51.17 & & 48.83 & $0.7153\left(\mathrm{sp}^{1.98}\right) \mathrm{C}+0.6988\left(\mathrm{sp}^{1.86}\right) \mathrm{C}$ & $\begin{array}{l}33.57 \\
34.91\end{array}$ & $\begin{array}{l}66.40 \\
65.05\end{array}$ \\
\hline$\Sigma(\mathrm{C} 13-\mathrm{C} 14)$ & 1.98056 & 50.21 & & 49.79 & $0.7086\left(\mathrm{sp}^{1.79}\right) \mathrm{C}+0.7056\left(\mathrm{sp}^{1.83}\right) \mathrm{C}$ & $\begin{array}{l}35.81 \\
35.30\end{array}$ & $\begin{array}{l}64.15 \\
64.66\end{array}$ \\
\hline$\Sigma(\mathrm{C} 14-\mathrm{C} 15)$ & 1.98102 & 50.08 & & 49.92 & $0.7076\left(\mathrm{sp}^{1.85}\right) \mathrm{C}+0.7066\left(\mathrm{sp}^{1.86}\right) \mathrm{C}$ & $\begin{array}{l}35.01 \\
35.01\end{array}$ & $\begin{array}{l}64.95 \\
64.95\end{array}$ \\
\hline$\Sigma(\mathrm{C} 15-\mathrm{C} 16)$ & 1.98107 & 49.96 & & 50.04 & $0.7068\left(\mathrm{sp}^{1.84}\right) \mathrm{C}+0.7074\left(\mathrm{sp}^{1.85}\right) \mathrm{C}$ & $\begin{array}{l}35.19 \\
35.06\end{array}$ & $\begin{array}{l}64.77 \\
64.90\end{array}$ \\
\hline$\Sigma(\mathrm{C} 16-\mathrm{C} 17)$ & 1.98083 & 49.84 & & 50.16 & $0.7059\left(\mathrm{sp}^{1.84}\right) \mathrm{C}+0.7083\left(\mathrm{sp}^{1.81}\right) \mathrm{C}$ & $\begin{array}{l}35.15 \\
35.62\end{array}$ & $\begin{array}{l}64.81 \\
64.34\end{array}$ \\
\hline$\Sigma(\mathrm{N} 1-\mathrm{O} 1)$ & 1.99579 & 48.58 & & 51.42 & $0.6970\left(\mathrm{sp}^{2.13}\right) \mathrm{N}+0.7171\left(\mathrm{sp}^{2.97}\right) \mathrm{O}$ & $\begin{array}{l}31.92 \\
25.14\end{array}$ & $\begin{array}{l}67.98 \\
74.71\end{array}$ \\
\hline$\Sigma(\mathrm{N} 1-\mathrm{O} 2)$ & 1.99578 & 48.58 & & 51.42 & $0.6970\left(\mathrm{sp}^{2.13}\right) \mathrm{N}+0.7171\left(\mathrm{sp}^{2.98}\right) \mathrm{O}$ & $\begin{array}{l}31.89 \\
25.12\end{array}$ & $\begin{array}{l}68.01 \\
74.74\end{array}$ \\
\hline$\Pi(\mathrm{C} 1-\mathrm{C} 2)$ & 1.60465 & 52.47 & & 47.53 & $0.7244\left(\mathrm{sp}^{1.00}\right) \mathrm{C}+0.6894\left(\mathrm{sp}^{1.00}\right) \mathrm{C}$ & $\begin{array}{l}0.00 \\
0.00\end{array}$ & $\begin{array}{l}99.97 \\
99.95\end{array}$ \\
\hline П(C3-C4) & 1.64122 & 54.84 & & 45.16 & $0.7405\left(\mathrm{sp}^{1.00}\right) \mathrm{C}+0.6720\left(\mathrm{sp}^{1.00}\right) \mathrm{C}$ & $\begin{array}{l}0.00 \\
0.00\end{array}$ & $\begin{array}{l}99.99 \\
99.95\end{array}$ \\
\hline П(C5-C6) & 1.65472 & 51.48 & & 48.52 & $0.7175\left(\mathrm{sp}^{1.00}\right) \mathrm{C}+0.6965\left(\mathrm{sp}^{1.00}\right) \mathrm{C}$ & $\begin{array}{l}0.00 \\
0.00\end{array}$ & $\begin{array}{l}99.96 \\
99.96\end{array}$ \\
\hline П(C7-C8) & 1.84165 & 46.94 & & 53.06 & $0.6851\left(\mathrm{sp}^{1.00}\right) \mathrm{C}+0.7284\left(\mathrm{sp}^{1.00}\right) \mathrm{C}$ & $\begin{array}{l}0.00 \\
0.00\end{array}$ & $\begin{array}{l}99.94 \\
99.96\end{array}$ \\
\hline П(C9-O3) & 1.96464 & 32.49 & & 67.51 & $0.5700\left(\mathrm{sp}^{1.00}\right) \mathrm{C}+0.8217\left(\mathrm{sp}^{1.00}\right) \mathrm{O}$ & $\begin{array}{l}0.00 \\
0.00\end{array}$ & $\begin{array}{l}99.85 \\
99.70\end{array}$ \\
\hline$\Pi(\mathrm{C} 10-\mathrm{C} 11)$ & 1.83189 & 54.56 & & 45.44 & $0.7386\left(\mathrm{sp}^{1.00}\right) \mathrm{C}+0.6741\left(\mathrm{sp}^{1.00}\right) \mathrm{C}$ & $\begin{array}{l}0.00 \\
0.00\end{array}$ & $\begin{array}{l}99.96 \\
99.94\end{array}$ \\
\hline П(C12-C17) & 1.61495 & 51.56 & & 48.44 & $0.7180\left(\mathrm{sp}^{1.00}\right) \mathrm{C}+0.6960\left(\mathrm{sp}^{1.00}\right) \mathrm{C}$ & $\begin{array}{l}0.00 \\
0.00\end{array}$ & $\begin{array}{l}99.98 \\
99.96\end{array}$ \\
\hline П(C13-C14) & 1.67848 & 49.46 & & 50.54 & $0.7033\left(\mathrm{sp}^{1.00}\right) \mathrm{C}+0.7109\left(\mathrm{sp}^{1.00}\right) \mathrm{C}$ & $\begin{array}{l}0.00 \\
0.00\end{array}$ & $\begin{array}{l}99.96 \\
99.96\end{array}$ \\
\hline$\Pi(\mathrm{C} 15-\mathrm{C} 16)$ & 1.65023 & 49.43 & & 50.57 & $0.7030\left(\mathrm{sp}^{1.00}\right) \mathrm{C}+0.7112\left(\mathrm{sp}^{1.00}\right) \mathrm{C}$ & $\begin{array}{l}0.00 \\
0.00\end{array}$ & $\begin{array}{l}99.96 \\
99.96\end{array}$ \\
\hline П(N1-O1) & 1.98600 & 40.04 & & 59.96 & $0.6327\left(\mathrm{sp}^{1.00}\right) \mathrm{N}+0.7744\left(\mathrm{sp}^{1.00}\right) \mathrm{O}$ & $\begin{array}{l}0.00 \\
0.00\end{array}$ & $\begin{array}{l}99.73 \\
99.75\end{array}$ \\
\hline $\mathrm{n} 1(\mathrm{O} 1)$ & 1.98113 & & & & $\mathrm{sp}^{0.34}$ & 74.83 & 25.16 \\
\hline $\mathrm{n} 2(\mathrm{O} 1)$ & 1.89710 & & & & & 0.14 & 99.76 \\
\hline $\mathrm{n} 1(\mathrm{O} 2)$ & 1.98117 & & & & & 74.86 & 25.14 \\
\hline $\mathrm{n} 2(\mathrm{O} 2)$ & 1.89771 & & & & & 0.14 & 99.76 \\
\hline n3(O2) & 1.44242 & & & & & 0.00 & 99.78 \\
\hline n1(O3) & 1.97822 & & & & & 58.20 & 41.76 \\
\hline n2(O3) & 1.88050 & - & - & & & 0.00 & 99.80 \\
\hline$\sigma^{*}(\mathrm{C} 1-\mathrm{C} 2)$ & 0.02070 & 49.35 & 50.65 & 0 . & $\left(\mathrm{sp}^{2.01}\right) \mathrm{C}-0.7117\left(\mathrm{sp}^{1.82}\right) \mathrm{C}$ & $\begin{array}{l}33.26 \\
35.41\end{array}$ & $\begin{array}{l}66.70 \\
64.55\end{array}$ \\
\hline$\sigma^{*}(\mathrm{C} 1-\mathrm{C} 6)$ & 0.02493 & 48.96 & 51.04 & 0 . & $\left(\mathrm{sp}^{1.95}\right) \mathrm{C}-0.7144\left(\mathrm{sp}^{1.88}\right) \mathrm{C}$ & $\begin{array}{l}33.87 \\
34.73 \\
\end{array}$ & $\begin{array}{l}66.09 \\
65.23 \\
\end{array}$ \\
\hline
\end{tabular}




\begin{tabular}{|c|c|c|c|c|c|c|}
\hline Bond $(\mathrm{AB})$ & ED/energy(a.u.) & EDA $(\%)$ & EDB $(\%)$ & $\mathrm{NBO}$ & $\mathrm{S}(\%)$ & $\mathrm{p}(\%)$ \\
\hline$\Sigma *(\mathrm{C} 1-\mathrm{C} 7)$ & 0.02262 & 48.48 & 51.52 & $0.6963\left(\mathrm{sp}^{2.05}\right) \mathrm{C}-0.7178\left(\mathrm{sp}^{1.96}\right) \mathrm{C}$ & $\begin{array}{l}32.82 \\
33.75\end{array}$ & $\begin{array}{l}67.14 \\
66.20\end{array}$ \\
\hline$\Sigma *(\mathrm{C} 2-\mathrm{C} 3)$ & 0.02039 & 50.94 & 49.06 & $0.7137\left(\mathrm{sp}^{1.94}\right) \mathrm{C}-0.7004\left(\mathrm{sp}^{1.64}\right) \mathrm{C}$ & $\begin{array}{l}34.05 \\
37.90\end{array}$ & $\begin{array}{l}65.90 \\
62.07\end{array}$ \\
\hline$\Sigma^{*}(\mathrm{C} 3-\mathrm{C} 4)$ & 0.02198 & 48.85 & 51.15 & $0.6989\left(\mathrm{sp}^{1.65}\right) \mathrm{C}-0.7152\left(\mathrm{sp}^{1.97}\right) \mathrm{C}$ & $\begin{array}{l}37.77 \\
33.61\end{array}$ & $\begin{array}{l}62.19 \\
66.34\end{array}$ \\
\hline$\Sigma^{*}(\mathrm{C} 4-\mathrm{C} 5)$ & 0.01565 & 49.63 & 50.37 & $0.7045\left(\mathrm{sp}^{1.83}\right) \mathrm{C}-0.7097\left(\mathrm{sp}^{1.88}\right) \mathrm{C}$ & $\begin{array}{l}35.38 \\
34.76\end{array}$ & $\begin{array}{l}64.59 \\
65.20\end{array}$ \\
\hline$\Sigma^{*}(\mathrm{C} 5-\mathrm{C} 6)$ & 0.01399 & 50.16 & 49.84 & $0.7082\left(\mathrm{sp}^{1.83}\right) \mathrm{C}-0.7060\left(\mathrm{sp}^{1.80}\right) \mathrm{C}$ & $\begin{array}{l}35.27 \\
35.70\end{array}$ & $\begin{array}{l}64.69 \\
64.27\end{array}$ \\
\hline$\Sigma^{*}(\mathrm{C} 7-\mathrm{C} 8)$ & 0.01221 & 49.67 & 50.33 & $0.7048\left(\mathrm{sp}^{1.64}\right) \mathrm{C}-0.7094\left(\mathrm{sp}^{1.65}\right) \mathrm{C}$ & $\begin{array}{l}37.86 \\
37.79\end{array}$ & $\begin{array}{l}62.11 \\
62.18\end{array}$ \\
\hline$\Sigma *(\mathrm{C} 8-\mathrm{C} 9)$ & 0.06002 & 48.40 & 51.60 & $0.6957\left(\mathrm{sp}^{2.08}\right) \mathrm{C}-0.7183\left(\mathrm{sp}^{1.91}\right) \mathrm{C}$ & $\begin{array}{l}32.41 \\
34.38\end{array}$ & $\begin{array}{l}67.54 \\
65.57\end{array}$ \\
\hline$\Sigma *(\mathrm{C} 9-\mathrm{C} 10)$ & 0.05765 & 51.28 & 48.72 & $0.7161\left(\mathrm{sp}^{1.84}\right) \mathrm{C}-0.6980\left(\mathrm{sp}^{2.08}\right) \mathrm{C}$ & $\begin{array}{l}35.24 \\
32.43\end{array}$ & $\begin{array}{l}64.71 \\
67.53\end{array}$ \\
\hline$\Sigma *(\mathrm{C} 9-\mathrm{O} 3)$ & 0.01094 & 65.34 & 34.66 & $0.8083\left(\mathrm{sp}^{2.30}\right) \mathrm{C}-0.5888\left(\mathrm{sp}^{1.39}\right) \mathrm{O}$ & $\begin{array}{l}30.30 \\
41.76\end{array}$ & $\begin{array}{l}69.59 \\
57.91\end{array}$ \\
\hline$\Sigma *(\mathrm{C} 10-\mathrm{C} 11)$ & 0.01248 & 50.21 & 49.79 & $0.7086\left(\mathrm{sp}^{1.65}\right) \mathrm{C}-0.7056\left(\mathrm{sp}^{1.65}\right) \mathrm{C}$ & $\begin{array}{l}37.73 \\
37.69\end{array}$ & $\begin{array}{l}62.23 \\
62.27\end{array}$ \\
\hline$\Sigma^{*}(\mathrm{C} 11-\mathrm{C} 12)$ & 0.02243 & 51.06 & 48.94 & $0.7146\left(\mathrm{sp}^{1.92}\right) \mathrm{C}-0.6995\left(\mathrm{sp}^{2.08}\right) \mathrm{C}$ & $\begin{array}{l}34.29 \\
32.42\end{array}$ & $\begin{array}{l}65.67 \\
67.54\end{array}$ \\
\hline$\Sigma *(\mathrm{C} 12-\mathrm{C} 13)$ & 0.02492 & 48.92 & 51.08 & $0.6994\left(\mathrm{sp}^{1.94}\right) \mathrm{C}-0.7147\left(\mathrm{sp}^{1.88}\right) \mathrm{C}$ & $\begin{array}{l}33.97 \\
34.71\end{array}$ & $\begin{array}{l}65.99 \\
65.25\end{array}$ \\
\hline$\Sigma *(\mathrm{C} 12-\mathrm{C} 17)$ & 0.02104 & 48.83 & 51.17 & $0.6988\left(\mathrm{sp}^{1.98}\right) \mathrm{C}-0.7153\left(\mathrm{sp}^{1.86}\right) \mathrm{C}$ & $\begin{array}{l}33.57 \\
34.91\end{array}$ & $\begin{array}{l}66.40 \\
65.05\end{array}$ \\
\hline$\Sigma *(\mathrm{C} 13-\mathrm{C} 14)$ & 0.01407 & 49.79 & 50.21 & $0.7056\left(\mathrm{sp}^{1.79}\right) \mathrm{C}-0.7086\left(\mathrm{sp}^{1.83}\right) \mathrm{C}$ & $\begin{array}{l}35.81 \\
35.30\end{array}$ & $\begin{array}{l}64.15 \\
64.66\end{array}$ \\
\hline$\Sigma *(\mathrm{C} 14-\mathrm{C} 15)$ & 0.01583 & 49.92 & 50.08 & $0.7066\left(\mathrm{sp}^{1.85}\right) \mathrm{C}-0.7076\left(\mathrm{sp}^{1.86}\right) \mathrm{C}$ & $\begin{array}{l}35.01 \\
35.01\end{array}$ & $\begin{array}{l}64.95 \\
64.95\end{array}$ \\
\hline$\Sigma^{*}(\mathrm{C} 15-\mathrm{C} 16)$ & 0.01564 & 50.04 & 49.96 & $0.7074\left(\mathrm{sp}^{1.84}\right) \mathrm{C}-0.7068\left(\mathrm{sp}^{1.85}\right) \mathrm{C}$ & $\begin{array}{l}35.19 \\
35.06\end{array}$ & $\begin{array}{l}64.77 \\
64.90\end{array}$ \\
\hline$\Sigma^{*}(\mathrm{~N} 1-\mathrm{O} 1)$ & 0.05650 & 51.42 & 48.58 & $0.7171\left(\mathrm{sp}^{2.13}\right) \mathrm{N}-0.6970\left(\mathrm{sp}^{2.97}\right) \mathrm{O}$ & $\begin{array}{l}31.92 \\
25.14\end{array}$ & $\begin{array}{l}67.98 \\
74.71\end{array}$ \\
\hline$\Sigma^{*}(\mathrm{~N} 1-\mathrm{O} 2)$ & 0.05664 & 51.42 & 48.58 & $0.7171\left(\mathrm{sp}^{2.13}\right) \mathrm{N}-0.6970\left(\mathrm{sp}^{2.98}\right) \mathrm{O}$ & $\begin{array}{l}31.89 \\
25.12\end{array}$ & $\begin{array}{l}68.01 \\
74.74\end{array}$ \\
\hline$\Pi^{*}(\mathrm{C} 1-\mathrm{C} 2)$ & 0.34153 & 47.53 & 52.47 & $0.6894\left(\mathrm{sp}^{1.00}\right) \mathrm{C}-0.7244\left(\mathrm{sp}^{1.00}\right) \mathrm{C}$ & $\begin{array}{l}0.00 \\
0.00\end{array}$ & $\begin{array}{l}99.97 \\
99.95\end{array}$ \\
\hline$\Pi *(\mathrm{C} 3-\mathrm{C} 4)$ & 0.37340 & 45.16 & 54.84 & $0.6720\left(\mathrm{sp}^{1.00}\right) \mathrm{C}-0.7405\left(\mathrm{sp}^{1.00}\right) \mathrm{C}$ & $\begin{array}{l}0.00 \\
0.00\end{array}$ & $\begin{array}{l}99.99 \\
99.95\end{array}$ \\
\hline$\Pi^{*}(\mathrm{C} 5-\mathrm{C} 6)$ & 0.28810 & 48.52 & 51.48 & $0.6965\left(\mathrm{sp}^{1.00}\right) \mathrm{C}-0.7175\left(\mathrm{sp}^{1.00}\right) \mathrm{C}$ & $\begin{array}{l}0.00 \\
0.00\end{array}$ & $\begin{array}{l}99.96 \\
99.96\end{array}$ \\
\hline$\Pi^{*}(\mathrm{C} 7-\mathrm{C} 8)$ & 0.09427 & 53.06 & 46.94 & $0.7284\left(\mathrm{sp}^{1.00}\right) \mathrm{C}-0.6851\left(\mathrm{sp}^{1.00}\right) \mathrm{C}$ & $\begin{array}{l}0.00 \\
0.00\end{array}$ & $\begin{array}{l}99.94 \\
99.96\end{array}$ \\
\hline$\Pi *(\mathrm{C} 9-\mathrm{O} 3)$ & 0.23046 & 67.51 & 32.49 & $0.8217\left(\mathrm{sp}^{1.00}\right) \mathrm{C}-0.5700\left(\mathrm{sp}^{1.00}\right) \mathrm{O}$ & $\begin{array}{l}0.00 \\
0.00\end{array}$ & $\begin{array}{l}99.85 \\
99.70\end{array}$ \\
\hline$\Pi^{*}(\mathrm{C} 10-\mathrm{C} 11)$ & 0.10872 & 45.44 & 54.56 & $0.6741\left(\mathrm{sp}^{1.00}\right) \mathrm{C}-0.7386\left(\mathrm{sp}^{1.00}\right) \mathrm{C}$ & $\begin{array}{l}0.00 \\
0.00\end{array}$ & $\begin{array}{l}99.96 \\
99.94\end{array}$ \\
\hline$\Pi^{*}(\mathrm{C} 12-\mathrm{C} 17)$ & 0.37438 & 48.44 & 51.56 & $0.6960\left(\mathrm{sp}^{1.00}\right) \mathrm{C}-0.7180\left(\mathrm{sp}^{1.00}\right) \mathrm{C}$ & $\begin{array}{l}0.00 \\
0.00\end{array}$ & $\begin{array}{l}99.98 \\
99.96\end{array}$ \\
\hline$\Pi^{*}(\mathrm{C} 13-\mathrm{C} 14)$ & 0.29620 & 50.54 & 49.46 & $0.7109\left(\mathrm{sp}^{1.00}\right) \mathrm{C}-0.7033\left(\mathrm{sp}^{1.00}\right) \mathrm{C}$ & $\begin{array}{l}0.00 \\
0.00\end{array}$ & $\begin{array}{l}99.96 \\
99.96\end{array}$ \\
\hline$\Pi *(\mathrm{C} 15-\mathrm{C} 16)$ & 0.32266 & 50.57 & 49.43 & $0.7112\left(\mathrm{sp}^{1.00}\right) \mathrm{C}-0.7030\left(\mathrm{sp}^{1.00}\right) \mathrm{C}$ & $\begin{array}{l}0.00 \\
0.00\end{array}$ & $\begin{array}{l}99.96 \\
99.96\end{array}$ \\
\hline$\Pi^{*}(\mathrm{~N} 1-\mathrm{O} 1)$ & 0.62218 & 59.96 & 40.04 & $0.7744\left(\mathrm{sp}^{1.00}\right) \mathrm{N}-0.6327\left(\mathrm{sp}^{1.00}\right) \mathrm{O}$ & $\begin{array}{l}0.00 \\
0.00 \\
\end{array}$ & $\begin{array}{l}99.73 \\
99.75 \\
\end{array}$ \\
\hline
\end{tabular}

Table 4: The Molecular Electric Dipole Moment M (Debye), Polarizability $\mathrm{A}_{\text {tot }}\left(\times 10^{-23}\right.$ Esu) and First Order Hyperpolarizability $\mathrm{B}_{\text {tot }}\left(\times 10^{-30}\right.$ Esu) Values of Title Compound

\begin{tabular}{|c|c|c|c|}
\hline Parameters & B3LYP & Parameters & B3LYP \\
\hline$\mu_{\mathrm{x}}$ & -5.075 & $\beta_{\mathrm{xxx}}$ & -2452.638 \\
\hline$\mu_{\mathrm{y}}$ & 5.120 & $\beta_{\mathrm{xxy}}$ & -1540.676 \\
\hline$\mu_{\mathrm{z}}$ & 0.000 & $\beta_{\text {xyy }}$ & -25.275 \\
\hline$\mu(\mathrm{D})$ & 7.210 & $\beta_{\text {yyy }}$ & 59.765 \\
\hline$\alpha_{\mathrm{xx}}$ & 454.420 & $\beta_{\mathrm{xxz}}$ & -1.398 \\
\hline$\alpha_{\mathrm{xy}}$ & -3.548 & $\beta_{\mathrm{xyz}}$ & -0.173 \\
\hline$\alpha_{y y}$ & 200.126 & $\beta_{\text {yyz }}$ & -0.443 \\
\hline$\alpha_{\mathrm{xz}}$ & 0.005 & $\beta_{\mathrm{xzz}}$ & -1.200 \\
\hline$\alpha_{\mathrm{yz}}$ & -0.001 & $\beta_{\mathrm{yzz}}$ & 3.105 \\
\hline$\alpha_{\mathrm{zz}}$ & 64.036 & $\beta_{\mathrm{zzz}}$ & 0.992 \\
\hline$\alpha_{\text {tot }}(\mathrm{a} . \mathrm{u})$ & 239.528 & $\beta_{\text {tot }}($ a.u $)$ & 2886.158 \\
\hline$\alpha_{\text {tot }} \times 10^{-23}(\mathrm{esu})$ & 3.545 & $\beta_{\text {tot }} \times 10^{-30}(\mathrm{esu})$ & 24.936 \\
\hline
\end{tabular}


The intra-molecular interactions due to the orbital overlap of $\pi$ (C12-C17) over $\pi^{*}(\mathrm{C} 13-\mathrm{C} 14)$ and $\pi^{*}(\mathrm{C} 15-\mathrm{C} 16)$ with energies $19.18 \mathrm{kcal} / \mathrm{mol}$ and $19.60 \mathrm{kcal} / \mathrm{mol}, \pi(\mathrm{C} 13-\mathrm{C} 14)$ over $\pi^{*}(\mathrm{C} 12$ $\mathrm{C} 17)$ and $\pi^{*}(\mathrm{C} 15-\mathrm{C} 16)$ with stabilization energies $18.85 \mathrm{kcal} / \mathrm{mol}$ and $20.22 \mathrm{kcal} / \mathrm{mol}$ and $\pi(\mathrm{C} 15-\mathrm{C} 16)$ over $\pi^{*}(\mathrm{C} 12-\mathrm{C} 17)$ and $\pi^{*}$ $(\mathrm{C} 13-\mathrm{C} 14)$ with energies $21.18 \mathrm{kcal} / \mathrm{mol}$ and $18.97 \mathrm{kcal} / \mathrm{mol}$. these six transitions correspond to three pairs of orbitals within the second aromatic ring. Further the other high energy interactions involving the delocalization of $\pi^{*}$ electrons of aromatic with the anti-bonding $\pi^{*}$ electrons of aromatic corresponds to $\pi^{*}(\mathrm{C} 12$ $\mathrm{C} 17) \rightarrow \pi^{*}(\mathrm{C} 10-\mathrm{C} 11)$ and $\pi^{*}(\mathrm{C} 1-\mathrm{C} 2) / \pi^{*}(\mathrm{C} 7-\mathrm{C} 8)$ stabilizes the compound by stabilization energy in the range of $47.80107 .46 \mathrm{~kJ} / \mathrm{mol}$.

The large hyperconjugative interaction energies which are presented in Table 2 display the presence of intramolecular charge transfer (ICT) in title compound . It is well known that ICT induce the nonlinear optical properties of molecular systems.

The second order perturbation energies of significant donoracceptor interactions and occupancy of electrons and $\mathrm{p}-$-character in significant NBO natural atomic hybrid orbitals were presented in Table 3. Selected bond taken from Table 3, $\sigma(\mathrm{C} 9-\mathrm{O} 3)$ is composed of $0.5888\left(\mathrm{sp}^{2.30}\right) \mathrm{C}+0.8083\left(\mathrm{sp}^{1.39}\right) \mathrm{O}$. The numbers 0.5888 and 0.8083 represent the polarization co-efficient. The biggest value of the polarization coefficient, indicate higher value of the electronegativity. The weights are obtained from the squares of the coefficients as $(0.8083)^{2} \approx 0,65334889$, corresponding to $65.34 \%$ localization on oxygen atom. In a similar way, the $34.66 \%$ localization on carbon is obtained. The oxygen has larger percentage of this NBO, at $65.34 \%$ and gives the larger polarization coefficien of 0.8083 because it has higher electro negatively. In $\sigma$ (C3N1)orbital with 1.98966 electrons has $37.82 \% \mathrm{C} 3$ character in a sp2.06 hybrid and has $36.43 \%$ C9 character in asp s.12 $^{3 .}$ hybrid. The nitrogen has larger percentage of NBO at $62.18 \%$ and gives the larger polarization coefficient of 0.7885 becauseof its higher electro negativity.

\subsection{Frontier molecular orbital analysis}

The HOMOLUMO energy gap of the title compound was computed at DFT/B3LYP/6-31G (d, p) and DFT/ HSEH1PBE /6-31G $(d, p)$ levels. The electron density plots of the HOMO and LUMO for the title compound is presented in Fig. 2. The energy band gap is $3.7567 \mathrm{eV}$ from B3LYP and 3.4010 from HSEH1PBE shown in Fig. 2. The small value of band gap reflects the chemical activity of the compound and encourages the application of the title compound as nonlinear optical materials.

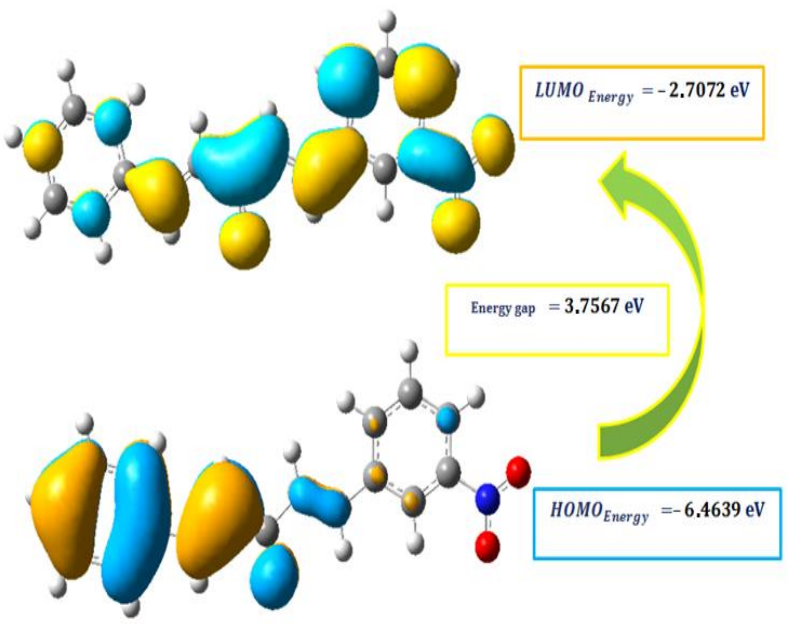

Fig. 2: The Molecular Orbitals and Energies for the HOMO and LUMO of the Title Compound.

\subsection{Molecular electrostatic potential (MESP) analysis}

Electrostatic potential in the MEP plot is increasing in the order of red $<$ orange $<$ yellow $<$ green $<$ blue. Electrostatic potential surface has been plotted for the title compound with B3LYP/6-31G $(\mathrm{d}, \mathrm{p})$ basis set using the computer software Gauss view as shown in Fig. 3. The negative regions are mainly localized on the nitro group and oxygen atom, a maximum positive region is localized on the hydrogen atoms indicating a possible site for nucleophilic attack.

\subsection{Nonlinear optical properties}

The non-linear optic properties (NLO) of the title compound was studied by calculating the dipole moment, polarizability and first order polarizability using B3LYP and HSEh1PBE methods with 6-31G $(\mathrm{d}, \mathrm{p})$ basis set. The NLO properties are summarized in Table 4.

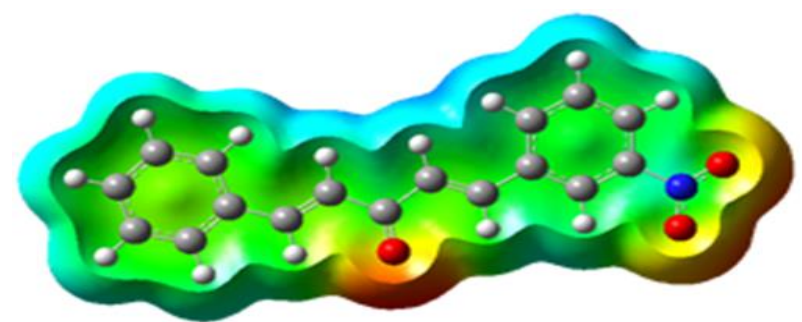

Fig. 3: Molecular Electrostatic Potential Map Obtained with B3LYP/6$31 \mathrm{G}(\mathrm{D}, \mathrm{P})$ Level.

The calculated molecular dipole moment 7.2100 and 7.1500D for B3LYP and HSEh1PBE levels, respectively. The calculated molecular polarizability $(\alpha)$ values equal $35.498 \times 10^{-23}$ and $35.093 \times$ $10^{-23}$ (e.s.u) for B3LYP and HSEh1PBE levels, respectively .The first order static hyperpolarizability $(\beta)$ parameter has been calculated as $24.936 \times 10^{-30}$ and $23.9523 \times 10^{-30}$ (e.s.u.) respectively. Our title compound with greater dipole moment and hyperpolarizability value shows that the compound has large NLO optical property.

\subsection{Vibrational assignments}

The title compound consists of 34 atoms, which has 96 normal modes of vibration. The vibrational band assignments were made by the aid of potential energy distribution (PED) using VEDA program. Vibrational frequency assignments of the title compound are reported in Table 5. The calculated infrared spectra of the title compound are shown in Fig. 4. Generally, the aromatic structure shows the presence of $\mathrm{CH}$ stretching vibration in the region 3100 $3000 \mathrm{~cm}^{1}$ (Silverstein et al. 1981, Wade 1992, Rastogi et al. 2002). In this present study, the $\mathrm{C}-\mathrm{H}$ stretching vibrations are observed at 3074 to $3023 \mathrm{~cm}^{-1}$ [mode number 96 to 90 ] by B3LYP/6-31G $(\mathrm{d}, \mathrm{P})$ method. The PED corresponding to this pure mode of title compound contributed $86 \%$ to $99 \%$. The $\mathrm{C}-\mathrm{C}-\mathrm{H}$ in plane bending vibrations are normally occurred as a number of strong to weak intensity bands in the region $1300-1000 \mathrm{~cm}^{-1}$. The theoretical wave numbers for B3LYP level at 1456, 1443, 1411, 1166, 1143, 1134,1126 and $1058 \mathrm{~cm}^{-1}$ (mode no 75, 74, 73, 61, 60, 59, 58, 56) are assigned as $\mathrm{C}-\mathrm{C}$ stretching vibrations with $\mathrm{PED}$ contributions of $60,52,46,34,76,64,79$ and $21 \%$, respectively. The $\mathrm{C}-\mathrm{H}$ outof-plane bending vibrations are strongly coupled vibrations and occur in the region $1000-750 \mathrm{~cm}^{-1}$.

The $\mathrm{C}-\mathrm{C}$ stretching vibrations of aromatic compounds are expected in the range from 1650 to $1200 \mathrm{~cm}^{-1}$. The theoretical wave numbers for B3LYP level at 1585,1570, 1545, 1541, 1533, 1391, 1306, 1052, 1296, 1052and 998 $\mathrm{cm}^{-1}$ (mode no 81, 80, 78, 77, 76, $72,65,55,69,53)$ are assigned as $\mathrm{C}-\mathrm{C}$ stretching vibrations with PED contributions of $61,36,69,58,41,45,40,53,31$ and $69 \%$, respectively. The $\mathrm{C}=\mathrm{O}$ stretching modes are expected around 1600 to $850 \mathrm{~cm}^{-1}$. The $\mathrm{C}=\mathrm{O}$ stretching vibrations are computed at 1659 c N, stretching; $\delta$, Scissoring; $\rho$, rooking; $\beta$, out-of-plane defor- 
mation; $\omega$, wagging; $\tau$, twisting; s, symmetric; as, antisymmetric.

M-1 for B3LYP level, and $1679 \mathrm{~cm}^{-1}$ for HSEh1PBE levels.

Percentage PED analysis is given in the brackets and PED

contribution less than $10 \%$ is neglected.

Table 5: Vibrational Frequency Assignments of the Title Compound

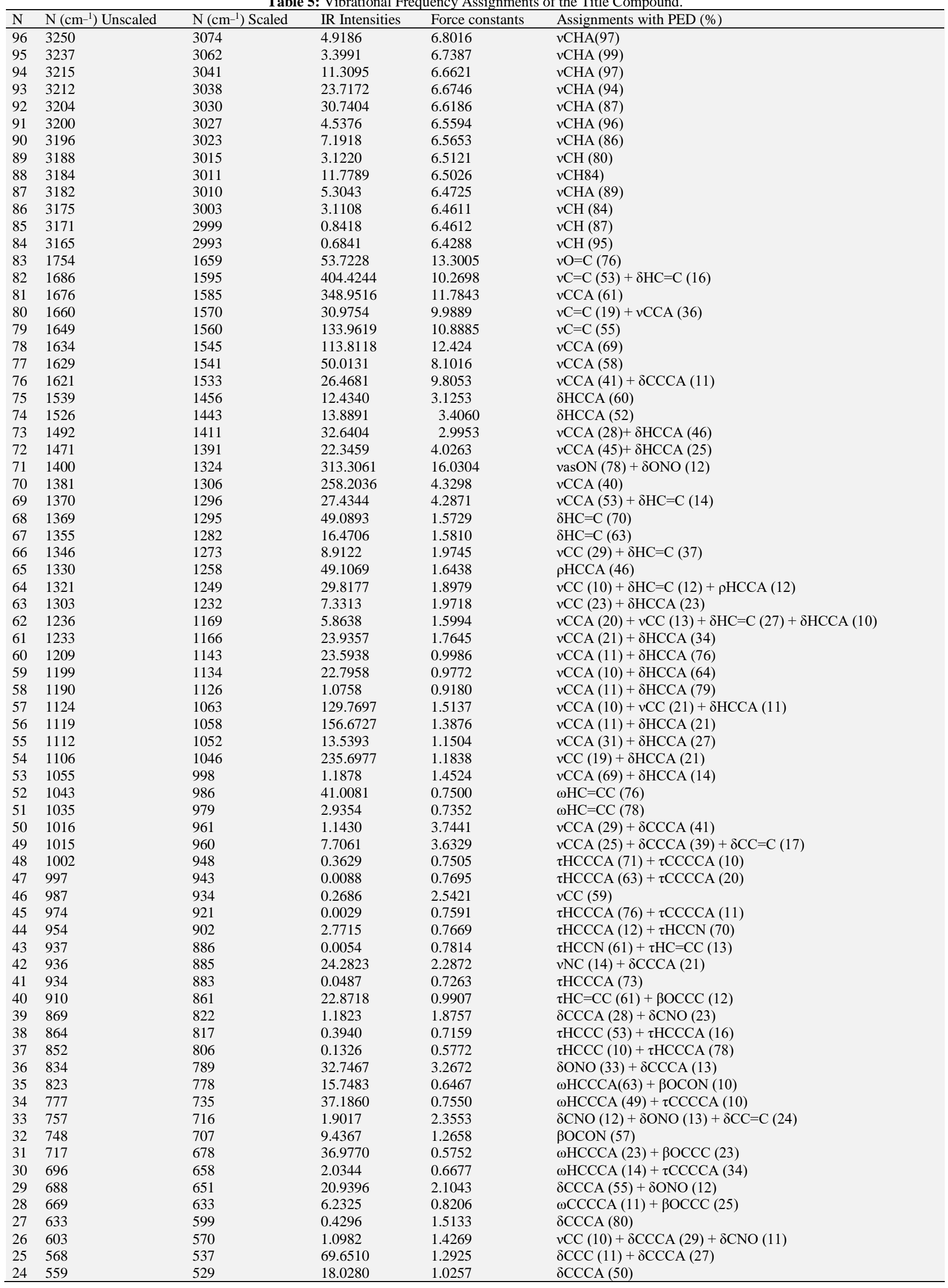




$\begin{array}{llllll}23 & 528 & 499 & 2.5960 & 0.6748 & \tau C C C A\end{array}$

Table 5: (Continued)

\begin{tabular}{|c|c|c|c|c|c|}
\hline $\mathrm{N}$ & $\mathrm{N}\left(\mathrm{cm}^{-1}\right)$ Unscaled & $\mathrm{N}\left(\mathrm{cm}^{-1}\right)$ Scaled & IR Intensities & Force constants & Assignments with PED (\%) \\
\hline 22 & 515 & 487 & 0.7856 & 0.8282 & $\delta \mathrm{CNO}(66)$ \\
\hline 21 & 498 & 471 & 7.3447 & 0.4217 & $\beta \mathrm{CCCC}(63)$ \\
\hline 20 & 438 & 414 & 0.0543 & 0.3713 & $\beta C C C C(13)+\tau \operatorname{CCCCA~}(54)$ \\
\hline 19 & 427 & 404 & 5.0972 & 0.6730 & $v \mathrm{NC}(16)+\delta \mathrm{CCC}(27)$ \\
\hline 18 & 413 & 391 & 0.0202 & 0.2922 & $\tau \operatorname{HCCCA}(21)+\tau \mathrm{CCCCA}(73)$ \\
\hline 17 & 379 & 358 & 6.1811 & 0.6139 & $v \mathrm{NC}(29)+\delta \mathrm{ONO}(12)+\delta \mathrm{CCCA}(11)$ \\
\hline 16 & 294 & 278 & 0.6106 & 0.3420 & $\delta \mathrm{CC}=\mathrm{C}(62)$ \\
\hline 15 & 288 & 272 & 1.6970 & 0.1522 & $\tau \mathrm{CCCCA}(55)$ \\
\hline 13 & 272 & 257 & 4.2743 & 0.3510 & $\delta \mathrm{CCCA}(65)$ \\
\hline 12 & 198 & 187 & 2.4883 & 0.1607 & $\delta \mathrm{CCC}(51)$ \\
\hline 11 & 187 & 177 & 0.0001 & 0.0511 & $\tau \mathrm{C}=\mathrm{CCC}(77)$ \\
\hline 10 & 173 & 164 & 1.2501 & 0.0894 & $\tau \mathrm{CCCCA}(77)$ \\
\hline 09 & 165 & 156 & 0.2678 & 0.1090 & $v C C(32)+\delta C C=C(37)$ \\
\hline 08 & 104 & 98 & 0.9337 & 0.0336 & $\tau \mathrm{C}=\mathrm{CCC}(49)+\tau \mathrm{CCCCA}$ \\
\hline 07 & 94 & 89 & 0.6286 & 0.0347 & $\delta \mathrm{CC}=\mathrm{C}(80)$ \\
\hline 06 & 83 & 79 & 0.1244 & 0.0198 & $\tau \mathrm{CCCCA}(46)+\tau \mathrm{CC}=\mathrm{CC}(26)$ \\
\hline 04 & 48 & 45 & 0.6288 & 0.0179 & $\tau \operatorname{CCCCA~}(72)$ \\
\hline 03 & 33 & 31 & 0.1215 & 0.0042 & $\delta \mathrm{CC}=\mathrm{C}(76)$ \\
\hline 02 & 20 & 19 & 0.4246 & 0.0011 & $\tau \mathrm{C}=\mathrm{CCC}(11)+\tau \mathrm{CCCCA}(62)$ \\
\hline 01 & 12 & 11 & 0.0476 & 0.0007 & $\tau \operatorname{CCCCA~}(80)$ \\
\hline
\end{tabular}

Table 6: Thermo Dynamical Parameters of Title Compound Calculated at B3LYP/6-31G (D, P)

\begin{tabular}{llll}
\hline Parameters & Value & Parameters & \\
\hline$\mu(\mathrm{D})$ & 7.21 & HOMO $(\mathrm{EV})$ & -6.46 \\
$\mathrm{I}$ & 6.46 & LUMO $(\mathrm{eV})$ & -2.71 \\
$\mathrm{~A}$ & 2.71 & $\Delta \mathrm{E}(\mathrm{eV})$ & 3.76 \\
$\chi$ & 4.59 & Zero-point vibrational energy $(\mathrm{J} / \mathrm{Mol})$ & 935.70 \\
$\mu$ & 4.59 & Zero-point correction (hatred/particle) & 0.26 \\
$\boldsymbol{\eta}$ & 1.88 & Thermal correction to energy & 0.28 \\
$\mathrm{~S}$ & 0.94 & Thermal correction to enthalpy & 0.28 \\
$\omega$ & 5.60 & Thermal correction to Gibbs free Ennery & 0.21 \\
\hline
\end{tabular}

Table 7: Calculated Absorption Wavelength $\Lambda(\mathrm{Nm})$, Excitation Energies E (Ev) and Oscillator Strengths (F) of the Title Compound Using TDDFT/B3LYP/6-31G (D, P) Basis Set.

\begin{tabular}{lll}
\hline Wavelength $\lambda(\mathrm{nm})$ & 361.88 & 333.68 \\
\hline Excitation energy $(\mathrm{eV})$ & 3.4261 & 3.7157 \\
Oscillator strengths (f) & 0.4519 & 0.2275 \\
& & H-2 $\rightarrow$ LUMO $(13 \%)$ \\
Major contributions & HOMO $\rightarrow$ LUMO (97\%) & H-2 $\rightarrow$ L+1 $(27 \%)$ \\
& & HOMO $\rightarrow$ L+1 $(55 \%)$ \\
Minor contribution & H-2 $\rightarrow$ LUMO (2\%) & H-4 $\rightarrow$ LUMO (2\%) \\
\hline
\end{tabular}

\begin{tabular}{|c|c|c|c|c|c|c|c|}
\hline Atoms & Mulliken & NPA & APT & Atoms & Mulliken & NPA & APT \\
\hline $\mathrm{C} 1$ & 0.110 & -0.075 & -0.017 & N1 & 0.391 & 0.514 & 1.237 \\
\hline $\mathrm{C} 2$ & -0.131 & -0.185 & -0.030 & $\mathrm{O} 1$ & -0.392 & -0.378 & -0.650 \\
\hline $\mathrm{C} 4$ & -0.083 & -0.206 & -0.057 & $\mathrm{O} 3$ & -0.500 & -0.570 & -0.859 \\
\hline C5 & -0.101 & -0.231 & -0.078 & $\mathrm{H} 2$ & 0.144 & 0.276 & 0.109 \\
\hline C6 & -0.089 & -0.178 & -0.019 & $\mathrm{H} 4$ & 0.141 & 0.276 & 0.105 \\
\hline C7 & -0.082 & -0.156 & 0.300 & H5 & 0.108 & 0.253 & 0.027 \\
\hline $\mathrm{C} 8$ & -0.146 & -0.292 & -0.599 & H6 & 0.099 & 0.243 & 0.046 \\
\hline C9 & 0.408 & 0.493 & 1.527 & $\mathrm{H} 7$ & 0.129 & 0.259 & 0.064 \\
\hline $\mathrm{C} 10$ & -0.146 & -0.316 & -0.757 & H8 & 0.081 & 0.222 & 0.027 \\
\hline C11 & -0.090 & -0.134 & 0.491 & H10 & 0.079 & 0.222 & 0.026 \\
\hline $\mathrm{C} 12$ & 0.127 & -0.089 & -0.145 & H11 & 0.122 & 0.253 & 0.057 \\
\hline C13 & -0.108 & -0.199 & -0.028 & H13 & 0.086 & 0.236 & 0.034 \\
\hline C14 & -0.089 & -0.235 & -0.070 & H14 & 0.093 & 0.243 & 0.015 \\
\hline C15 & -0.078 & -0.220 & -0.022 & H15 & 0.094 & 0.243 & 0.018 \\
\hline C16 & -0.088 & -0.236 & -0.027 & H16 & 0.095 & 0.245 & 0.013 \\
\hline
\end{tabular}

The $\mathrm{C}=\mathrm{C}$ stretching modes are expected around 1650 to $1100 \mathrm{~cm}-$ 1 (Clothup et al. 1990, Roeges 1994) when conjugated with a carbonyl group (Socrates 1981). The carbonyl stretching vibrations appear in the region $1750-1600 \mathrm{~cm} 1$. In the present case, the band calculated at $1659 \mathrm{~cm} 1$ with PED contribution of $76 \%$ was assigned as the carbonyl stretching vibration, which are in well agreement with the reported values for chalcone derivatives (Alwani Zainuri et al. 2017). The NO2 group compounds are readily identified by asymmetric and symmetric stretching bands. The asymmetric NO2 stretching vibrations are generally observed in the region $1570-1485 \mathrm{~cm}-1$, while the symmetric stretch will appear between 1370-1320 cm-1 (Meislich et al. 1993). In the present compound, the strong band at $1400 \mathrm{~cm}-1$ by DFT method has been assigned to asymmetric stretching mode of $\mathrm{N}-\mathrm{O}$. 


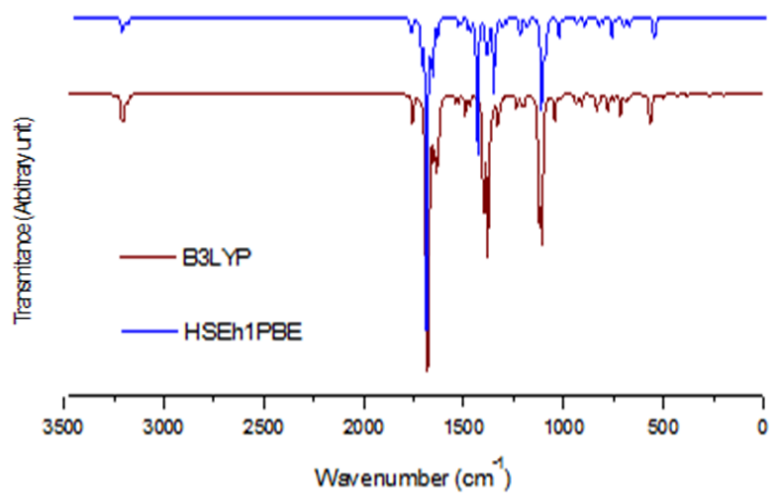

Fig. 4: The Calculated Infrared Spectra of the Title Compound.

\subsection{Global reactivity descriptors and thermodynamic parameters}

Energies of frontier molecular orbitals (Еномо, Elumo), have been used to calculate global chemical reactivity descriptors of compounds such as ionization potential, electron affinity, electronegativity, Global hardness, global softness, chemical potential and electrophilicity index. The calculated HOMO-LUMO energies, Energy gap are presented in Table 6 . The Ionization potential (I) and an electron affinity (A) of the title compound calculated by B3LYP and HSEh1PBE levels are $6.4639,6.3551 \mathrm{eV}$ and $2.7072,2.9543 \mathrm{eV}$ respectively. The calculated values of the hardness, softness, chemical potential, electronegativity and electrophilicity index of our compound at B3LYP are 1,8784, $0,9392,-4,5855,4,5855$ and 5,5973 respectively as shown in Table 6. It is seen that the chemical potential of title compound is negative which means that these substances are stable. The values of some thermodynamic parameters of the title compound calculated using B3LYP/6-and 31G (d,p) HSEH1PBE/6-31G (d,p) levels are summarized in Table 6.

\subsection{Electronic properties and UV spectra}

In order to recognize electronic transitions of the title compound, calculations in gas phase were performed by using TDDFT/B3LYP/631G (d,p) for six excited states. The calculated absorption wavelengths $(\lambda)$, oscillator strengths (f), and excitation energies (E) are given Table 7. The theoretical UV-Vis spectra of the title compound are shown in Fig 5. The calculated value at $361.88 \mathrm{~nm}$ is attributed to $\mathrm{HOMO} \rightarrow$ LUMO electronic transition with $97 \%$ contribution. The electronic transitions is due to $\mathrm{n} \rightarrow \pi^{*}$ transition and may be attributed to the excitation of $\mathrm{C}=\mathrm{O}$ group. Gauss-Sum 2.2 program was used to calculate group contributions to the molecular orbitals and prepare the density of states spectrum in Fig. 5.

\subsection{Mulliken population analysis and natural popula- tion analysis}

The calculated Mulliken charge values are listed in Table 8 . The better represented graphical forms of our results were depicted in Fig. 6. The magnitude of the carbon atomic charge is found to be positive and negative. The magnitude of the hydrogen atomic charges is found to be only positive and is arranged in an order from 0.079012 to 0.144755 for title compound. The maximum atomic charge is obtained for $\mathrm{C} 9$ when compared with other atoms. This is due to the attachment of negatively charged carbon C9 atom. Negatively charged lone pair oxygen (O3) atom shows that charge is transferred from $\mathrm{C}$ to $\mathrm{O}(\mathrm{C} 9 \rightarrow \mathrm{O} 3)$.
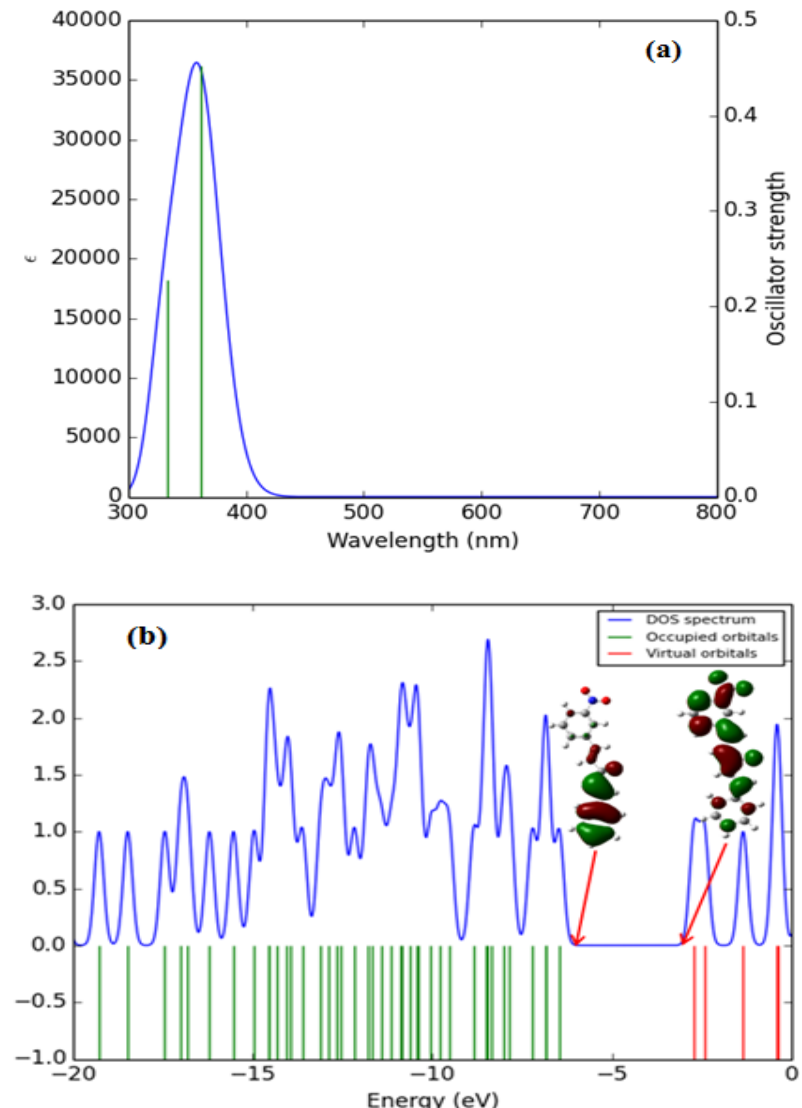

Fig. 5: Theoretical UV-Vis Spectrum (A) and Density of States (DOS) Diagram (B) of the Title Compound

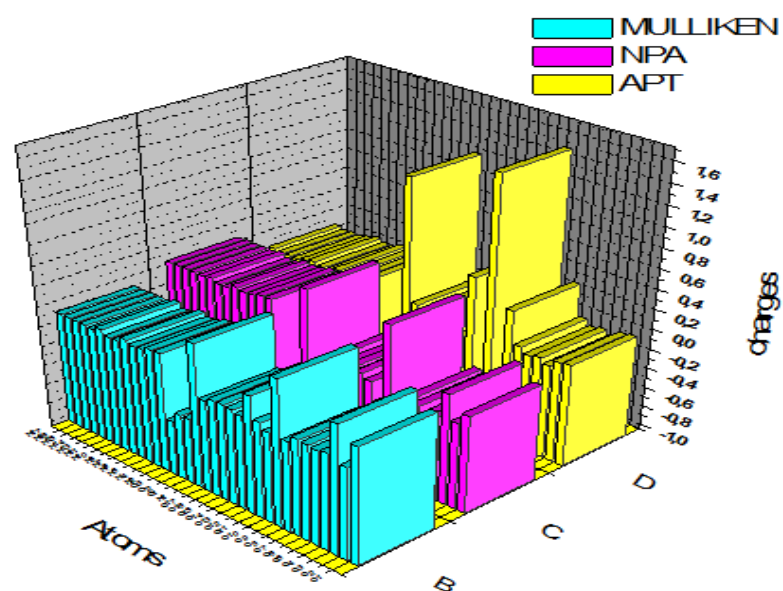

Fig. 6: The Mulliken Charges Diagram of the Title Compound

\section{Conclusion}

The optimized geometrical parameters of title compound were performed and analyzed using DFT calculation based on B3LYP and HSEH1PBE levels of theory with the standard basis set $6-$ $31 \mathrm{G}(\mathrm{d}, \mathrm{p})$. The vibrational wavenumbers, infrared intensities and force constants were calculated and a complete vibrational analysis of the title compound has been carried out. The dipole moment, polarizability and first order hyperpolarizability of the studied compound have been calculated by DFT method which indicated that the title compound is a material which has substantial nonlinear optical character. The frontier molecular orbitals have been visualized and the HOMO-LUMO energy gap has been calculated and can be used to estimate the ionization potential, electron affinity, electronegativity, electrophilicity index, global hardness, softness and chemical potential of the compound. Finally, all these results displayed that the title compound exhibits considerable NLO properties. 


\section{References}

[1] Abonia R, Insuasty D, Castillo J, Insuasty B, Quiroga J, Nogueras M \& Cobo J (2012), Synthesis of novel quinoline-2-one based chalcones of potential anti-tumor activity. European Journal of Medicinal Chemistry 57, 29-40. https://doi.org/10.1016/j.ejmech.2012.08.039.

[2] Zangade SB, Jadhav JD, Lalpod, Vibhute YB \& Dawane BS (2010), Synthesis and antimicrobial activity of some new chalcones and flavones containing substituted naphthalene moiety. Journal of Chemical and Pharmaceutical Research 2(1), 310-314.

[3] Tala-Tapeh SM, Mahmoodi N \& Vaziri A (2015) Synthesis of bischalcones based on 5, 5'-methylene bis(2-hydroxybenzaldehyde) and screening their antibacterial activity. Journal of Applied Chemistry 9(32), 53-58.

[4] Ballesteros JF, Sanz MJ, Ubeda A, Miranda MA, Iborra S, Paya M \& Alcarz MJ (1995), Synthesis and Pharmacological Evaluation of 2'-Hydroxychalcones and Flavones as Inhibitors of Inflammatory Mediators Generation. Journal of Medicinal Chemistry 38(14), 2794-2797. https://doi.org/10.1021/jm00014a032.

[5] Won SJ, Liu CT, Tsao LT, Weng JR, Ko HH, Wang JP \& Lin CN (2005), Synthetic chalcones as potential anti-inflammatory and cancer chemopreventive agents. European Journal of Medicinal Chemistry 40(1), 103-112. https://doi.org/10.1016/j.ejmech.2004.09.006.

[6] Kumar SK, Hager E, Pettit C, Gurulingappa H, Davidson NE \& Khan SR (2003), Design, synthesis, and evaluation of novel boronic-chalcone derivatives as antitumor agents. Journal of Medicinal Chemistry 46(14), 2813-2815. https://doi.org/10.1021/jm030213.

[7] Domínguez JN, León C, Rodrigues J, De Domínguez NG, Gut J \& Rosenthal PJ (2005), Synthesis and antimalarial activity of sulfonamide chalcone derivatives. Il Farmaco 60, 307-311. https://doi.org/10.1016/j.farmac.2005.01.005.

[8] Awasthi SK, Mishra N, Kumar B, Sharma M, Bhattacharya A, Mishra LC \& Bhasin VK (2009), Potent antimalarial activity of newly synthesized substituted chalcone analogs in vitro. Medicinal Chemistry Research 18(6), 407-420. https://doi.org/10.1007/s00044-008-9137-9.

[9] Rao YK, Fang SH \& Tzeng YM (2009), Synthesis and biological evaluation of 3', 4', 5'-trimethoxychalcone analogues as inhibitors of nitric oxide production and tumor cell proliferation. Bioorganic \& Medicinal Chemistry 17(23), 7909-7914 https://doi.org/10.1016/j.bmc.2009.10.022.

[10] Reddy MVB, Shen YC, Ohkoshi E, Bastow KF, Qian K, Lee KH \& Wu TS (2012), Bis-chalcone analogues as potent NO production inhibitors and as cytotoxic agents. European Journal of Medicinal Chemistry 47 ,

97-103. https://doi.org/10.1016/j.ejmech.2011.10.026.

[11] Zhao LM, Jin SH, Sun LP, Piao HR \& Quan ZS (2005), Synthesis and evaluation of antiplatelet activity of trihydroxychalcone derivatives. Bioorganic \& Medicinal Chemistry Letters 15(22), 5027 5029. https://doi.org/10.1016/j.bmcl.2005.08.039.

[12] Aponte JC, Castillo D, Estevez Y, Gonzalez G, Arevalo J, Hammond GB \& Sauvain M (2010), In vitro and in vivo antiLeishmania activity of polysubstituted synthetic chalcones. Bioorganic \& Medicinal Chemistry Letters 20(1), 100-103. https://doi.org/10.1016/j.bmcl.2009.11.033.

[13] Biradar JS, Sasidhar BS \& Parveen R (2010). Synthesis, antioxidant and DNA cleavage activities of novel indole derivatives. European Journal of Medicinal Chemistry 45, 4074-4078. https://doi.org/10.1016/j.ejmech.2010.05.067.

[14] Mizuno CS, Paul S, Suh N \& Rimando AM (2010), Synthesis and biological evaluation of retinoid-chalcones as inhibitors of colon cancer cell growth. Bioorganic \& Medicinal Chemistry Letters 20(24), 7385-7387. https://doi.org/10.1016/j.bmcl.2010.10.038

[15] Nabi G \& Liu ZQ (2011), Radical-scavenging properties of ferrocenyl chalcones. Bioorganic \& Medicinal Chemistry Letters 21(3), 944-946. https://doi.org/10.1016/j.bmcl.2010.12.051.

[16] Shukla P, Srivastava SP, Srivastava R, Rawat AK, Srivastava AK \& Pratap R (2011), Synthesis and antidyslipidemic activity of chalcone fibrates. Bioorganic \& Medicinal Chemistry Letters 21(11), 3475-3478. https://doi.org/10.1016/j.bmcl.2011.03.057.

[17] Hsieh CT, Hsieh TJ, El-Shazly M, Chuang DW, Tsai YH, Yen CT, Wu SF, Wu YC \& Chang FR (2012), Synthesis of chalcone derivatives as potential anti-diabetic agents. Bioorganic \& Medicinal Chemistry Letters 22(12), 3912-3915 https://doi.org/10.1016/j.bmcl.2012.04.108

[18] Dong X, Du L, Pan Z, Liu T, Yang B \& Hu Y (2010), Synthesis and biological evaluation of novel hybrid chalcone derivatives as vasorelaxant agents. European Journal of Medicinal Chemistry 45(9), 3986-3992. . https://doi.org/10.1016/j.ejmech.2010.05.054.

[19] Hayat F, Moseley E, Salahuddin A, Van Zyl RL \& Azam A (2011), antiprotozoal activity of chloroquinoline based chalcones. European Journal of Medicinal Chemistry 46(5) 1897-1905. https://doi.org/10.1016/j.ejmech.2011.02.004.

[20] Shettigar S, Umesh G, Chandrasekharan K \& Sarojini BK (2008), Studies on third-order nonlinear optical properties of chalcone derivatives in polymer host. Optical Materials 30(8), 1297-1303. https://doi.org/10.1016/i.optmat.2007.06.008.

[21] Asiri AM, Marwani HM, Alamry KA, Al-Amoudi MS, Khan SA \& El-Daly SA (2014), Green Synthesis, Characterization, Photophysical and Electrochemical Properties of Bis-chalcones. International Journal of Electrochemical Science 9, 799-809.

[22] Shettigar S, Chandrasekharan K, Umesh G \& Sarojini BK (2006), Studies on nonlinear optical parameters of bis-chalcone derivatives doped polymer. Polymer 47(10) 3565-3567. https://doi.org/10.1016/j.polymer.2006.03.062.

[23] Delavaux-Nicot B, Maynadié J \& Lavabre D (2007), Ca2+ vs. $\mathrm{Ba} 2+$ electrochemical detection by two disubstituted ferrocenyl chalcone chemosensors. Study of the ligand-metal interactions in CH3CN. Journal of Organometallic Chemistry 692(4), 874-886. https://doi.org/10.1016/j.jorganchem.2006.10.045.

[24] Gasull EI, Blanco SE \& Ferretti FH (2002), a theoretical and experimental study of adsorption from dilute cyclohexane solutions of non-electrolytes: 4-X-chalcones on silica gel. Journal of Molecular Structure: $\quad$ THEOCHEM 579(1-3), 121-137. https://doi.org/10.1016/S0166-1280(01)00723-0.

[25] Goto Y, Hayashi A, Kimura Y \& Nakayama M (1991), Second harmonic generation and crystal growth of substituted thienyl chalcone. Journal of Crystal Growth 108(3-4), 688-698. https://doi.org/10.1016/0022-0248(91)90249-5.

[26] Frisch MJ, et al., (2009), Gaussian 09, Revision B.01, Gaussian Inc., Wallingford CT.

[27] Becke AD (1993), Density-functional thermochemistry. III. The role of exact exchange. The Journal of Chemical Physics 98(7), 5648-5652. https://doi.org/10.1063/1.464913.

[28] Lee C, Yang W \& Parr RG (1988), Development of the ColleSalvetti correlation energy formula into a functional of the electron density. Physical Review B 37 (2), 785-789. https://doi.org/10.1103/PhysRevB.37.785.

[29] Heyd J \& Scuseria GE (2004), efficient hybrid density functional calculations in solids: Assessment of the Heyd-Scuseria-Ernzerho screened Coulomb hybrid functional. The Journal of Chemical Physics 121, 1187. https://doi.org/10.1063/1.1760074.

[30] Heyd J \& Scuseria G E (2004), Assessment and validation of a screened Coulomb hybrid density functional. The Journal of Chemical Physics 120, 7274. https://doi.org/10.1063/1.1668634.

[31] Frisch E, Hratchian HP, Dennington II RD, Keith TA, Millam J, Nielsen B, Holder AJ \& Hiscocks J (2009), Gaussian, Inc. Gauss View Version 5.0.8, Wallinford, CT.

[32] Jamroz MH (2004), Vibrational Energy Distribution Analysis: VEDA 4 Program, Warsaw, Poland.

[33] Glendening ED, Reed AE, Carpenter JE \& Weinhold F (1998), NBO Version3.1, TCI, University of Wisconsin, Madison.

[34] Foster JP \& Weinhold F (1980), Natural hybrid orbitals. Journal of the American Chemical Society 102(24), 7211-7218. https://doi.org/10.1021/ja00544a007.

[35] Reed AE \& Weinhold F (1983), Natural bond orbital analysis of near-Hartree-Fock water dimer. The Journal of Chemical Physics 78, 4066-4073. https://doi.org/10.1063/1.445134.

[36] Reed AE \& Weinhold F (1985), Natural localized molecular orbitals. The Journal of Chemical Physics 83, 1736-1740. https://doi.org/10.1063/1.449360.

[37] Reed AE, Weinstock RB \& Weinhold F (1985), Natural population analysis. The Journal of Chemical Physics 83, 735-746. https://doi.org/10.1063/1.449486.

[38] Reed AE, Curtiss LA \& Weinhold F (1988), Intermolecular interactions from a natural bond orbital, donor-acceptor viewpoint. Chemical Reviews 88(6), 899-926. https://doi.org/10.1021/cr00088a005.

[39] Szafran M, Komasa a \& Bartoszak-Adamska E (2007), Crystal and molecular structure of 4-carboxypiperidinium chloride (4piperidinecarboxylic acid hydrochloride). Journal of Molecular $\begin{array}{lll}\text { Structure } & 827(1-3), & 101-107 .\end{array}$ https://doi.org/10.1016/j.molstruc.2006.05.012.

[40] Sebastian S \& Sundaraganesan N (2010), the spectroscopic (FT-IR, FT-IR gas phase, FT-Raman and UV) and NBO analysis of 4Hydroxypiperidine by density functional method. Spectrochimica 
Acta Part A: Molecular and Biomolecular Spectroscopy 75(3), 941952. https://doi.org/10.1016/j.saa.2009.11.030.

[41] Tamer Ö, Avcı D \& Atalay Y (2015), Synthesis, X-ray structure, spectroscopic characterization and nonlinear optical properties of Nickel (II) complex with picolinate: A combined experimental and theoretical study. Journal of Molecular Structure 1098, 12-20. https://doi.org/10.1016/j.molstruc.2015.05.035.

[42] Altürk S, Tamer Ö, Avcı D \& Atalay Y (2015), Synthesis, spectroscopic characterization, second and third-order nonlinear optical properties, and DFT calculations of a novel Mn(II) complex. Journal of Organometallic Chemistry 797, 110-119. https://doi.org/10.1016/j.jorganchem.2015.08.014.

[43] Stratmann RE, Scuseria GE \& Frisch MJ (1998), an efficient implementation of time-dependent density-functional theory for the calculation of excitation energies of large molecules. The Journal of Chemical Physics 109, 8218-8224. https://doi.org/10.1063/1.477483.

[44] Cancès E, Mennucci B \& Tomasi J (1997), a new integral equation formalism for the polarizable continuum model: Theoretical background and applications to isotropic and anisotropic dielectrics. The Journal of Chemical Physics 107, 3032-3041. https://doi.org/10.1063/1.474659.

[45] O'Boyle NM, Tenderholt AL \& Langner KM (2008), cclib: a library for package-independent computational chemistry algorithms. Journal of Computational Chemistry 29(5), 839-845. https://doi.org/10.1002/jcc.20823.

[46] Ferreira MMC (1993), Population analysis from atomic polar tensors. Journal of Molecular Structure 294, 75-78. https://doi.org/10.1016/0022-2860(93)80318-P.

[47] Parr RG, Szentpály LV \& Liu S (1999), Electrophilicity Index. Journal of the American Chemical Society 121(9), 1922-1924. https://doi.org/10.1021/ja983494x.

[48] Chattaraj P.K., Maiti B \& Sarkar U (2003), Philicity: A Unified Treatment of Chemical Reactivity and Selectivity. Journal of Physical Chemistry a 107, 4973-4975. https://doi.org/10.1021/jp034707u.

[49] Parr RG, Donnelly RA, Levy M \& Palke WE (1978), Electronegativity: the density functional viewpoint. Journal of Chemical Physics 68(8), 3801-3807. https://doi.org/10.1063/1.436185.

[50] Parr RG \& Pearson RG (1983), Absolute hardness: companion parameter to absolute electronegativity. Journal of the American Chemical Society 105(26), 7512-7516. https://doi.org/10.1021/ja00364a005.

[51] Pearson RG (2005), Chemical hardness and density functional theory. Journal of Chemical Sciences 117(5), 369-377. https://doi.org/10.1007/BF02708340.

[52] Alwani Zainuri D, Arshad S, Che Khalib N, Abdul Razak I, Raveendran Pillai R, Farizaa Sulaiman S, Shafiqah Hashim N, Leong Ooi K, Armaković S, Armaković SJ, Yohannan Panicker C \& Van Alsenoy C (2017), Synthesis, XRD crystal structure, spectroscopic characterization (FT-IR, 1H and 13C NMR), DFT studies, chemical reactivity and bond dissociation energy studies using molecular dynamics simulations and evaluation of antimicrobial and antioxidant activities of a novel chalcone derivative, (E)-1-(4bromophenyl)-3-(4-iodophenyl)prop-2-en-1-one. Journal of Molecular Structure 1128, 520-533. https://doi.org/10.1016/j.molstruc.2016.09.022.

[53] Samshuddin S, Butcher RJ, Akkurt M, Narayana B, Sarojini BK \& Yathirajan HS (2012), (1E,4E)-1-(3-Nitro-phen-yl)-5-phenyl-penta1,4-dien-3-one. Acta Crystallographica Section E 68, o74-o75. https://doi.org/10.1107/S1600536811052548.

[54] Rastogi VK, Palafox MA, Tanwar RP \& Mittal L (2002), 3, 5Difluorobenzonitrile: ab initio calculations, FTIR and Raman spectra. Spectrochimica Acta Part A: Molecular and Biomolecular Spectroscopy 58(9), 1987-2004. https://doi.org/10.1016/S13861425(01)00650-3.

[55] Silverstein M, Basseler GC \& Morill C (1981), Spectrometric Identification of Organic Compounds, Wiley, New York.

[56] Wade LG (1992), Advanced Organic Chemistry, fourth ed., Wiley, New York.

[57] Roeges NPG (1994), a Guide to the Complete Interpretation of IR Spectra of Organic Compounds, Wiley, New York.

[58] Clothup NB, Daly LH \& Wiberly SE (1990), Introduction to IR and Raman Spectroscopy, Academic Press, New York.

[59] Socrates G (1981), Infrared Characteristic Group Frequencies, John Wiley and Sons, New York.

[60] Meislich EK, Meislich H \& Sharefkin J (1993), 3000 Solved Problems in Organic Chemistry, vol. 2, McGraw-Hill, New York. 